\title{
Spleen-derived classical monocytes mediate lung ischemia-reperfusion injury through IL-1及
}

\author{
Hsi-Min Hsiao, ${ }^{1}$ Ramiro Fernandez, ${ }^{2}$ Satona Tanaka, ${ }^{1}$ Wenjun Li, ${ }^{1}$ Jessica H. Spahn, ${ }^{1}$ Stephen Chiu, ${ }^{2}$ Mahzad Akbarpour, ${ }^{2}$ \\ Daniel Ruiz-Perez, ${ }^{1}$ Qiang Wu, ${ }^{2}$ Cem Turam, ${ }^{1}$ Davide Scozzi, ${ }^{1}$ Tsuyoshi Takahashi, ${ }^{1}$ Hannah P. Luehmann, ${ }^{3}$ Varun Puri, \\ G.R. Scott Budinger, ${ }^{4}$ Alexander S. Krupnick, ${ }^{5}$ Alexander V. Misharin, ${ }^{4}$ Kory J. Lavine, ${ }^{6}$ Yongjian Liu, ${ }^{3}$ Andrew E. Gelman, \\ Ankit Bharat, ${ }^{2,4}$ and Daniel Kreisel ${ }^{1,7}$ \\ 'Department of Surgery, Washington University in St. Louis, St. Louis, Missouri, USA. ²Department of Surgery, Northwestern University, Chicago, Illinois, USA. ${ }^{3}$ Department of Radiology, Washington \\ University in St. Louis, St. Louis, Missouri, USA. ${ }^{4}$ Department of Medicine, Northwestern University, Chicago, Illinois, USA. ${ }^{5}$ Department of Surgery, The University of Virginia, Charlottesville, Virginia, USA. \\ ${ }^{6}$ Department of Medicine, and ${ }^{7}$ Department of Pathology \& Immunology, Washington University in St. Louis, St. Louis, Missouri, USA.
}

\begin{abstract}
Ischemia-reperfusion injury, a form of sterile inflammation, is the leading risk factor for both short-term mortality following pulmonary transplantation and chronic lung allograft dysfunction. While it is well recognized that neutrophils are critical mediators of acute lung injury, processes that guide their entry into pulmonary tissue are not well understood. Here, we found that CCR2+ classical monocytes are necessary and sufficient for mediating extravasation of neutrophils into pulmonary tissue during ischemia-reperfusion injury following hilar clamping or lung transplantation. The classical monocytes were mobilized from the host spleen, and splenectomy attenuated the recruitment of classical monocytes as well as the entry of neutrophils into injured lung tissue, which was associated with improved graft function. Neutrophil extravasation was mediated by MyD88-dependent IL-1 $\beta$ production by graft-infiltrating classical monocytes, which downregulated the expression of the tight junction-associated protein ZO-2 in pulmonary vascular endothelial cells. Thus, we have uncovered a crucial role for classical monocytes, mobilized from the spleen, in mediating neutrophil extravasation, with potential implications for targeting of recipient classical monocytes to ameliorate pulmonary ischemia-reperfusion injury in the clinic.
\end{abstract}

\section{Introduction}

Ischemia-reperfusion injury (IRI) following solid organ transplantation is mediated by host neutrophils that, upon extravasation, initiate NETosis and induce allograft damage $(1,2)$. Following lung transplantation, IRI leads to primary graft dysfunction in over $50 \%$ of lung recipients, which is the predominant risk factor for early mortality as well as chronic rejection and thus late mortality (3-6). While depletion of neutrophils can ameliorate IRI, it can also compromise the pathogen-directed response of the host (7-11). Elucidating the mechanisms of neutrophil trafficking into the allograft is, therefore, crucial to developing clinically applicable therapies against IRI.

Blood monocytes can be classified into nonclassical and classical subtypes based on cell surface markers $(12,13)$. Classical monocytes are characterized by a high expression of the cell-surface molecule Ly6C and the presence of the chemokine receptor CCR2. They are the predominant monocytes found in the circulation, where they survey organ spaces and readily respond to inflammatory cues. Classical monocytes are generally regarded as "proinflammatory," as they are readily recruited to sites of infectious and sterile inflamma-

Authorship note: $\mathrm{HMH}$ and $\mathrm{RF}$ contributed equally to this work. $\mathrm{AB}$ and $\mathrm{DK}$ are co-senior authors.

Conflict of interest: YL, DK, and KJL have a pending US patent entitled "Compositions and methods for detecting CCR2 receptors" (application number 15/611,577).

Submitted: November 3, 2017; Accepted: April 4, 2018.

Reference information: J Clin Invest. 2018;128(7):2833-2847.

https://doi.org/10.1172/JCI98436. tion, where they elaborate proinflammatory cytokines and differentiate into macrophages and dendritic cells (13). Nonclassical monocytes, in contrast, lack expression of CCR2, but express low levels of Ly6C and high levels of the fractalkine receptor $\mathrm{CX}_{3} \mathrm{CR} 1$. These cells patrol the microvasculature in a $\mathrm{CX}_{3} \mathrm{CR} 1$-dependent manner, and although less is known about the development and function of nonclassical monocytes, recent studies have implicated this monocyte subtype in various inflammatory processes (14-21). The precise role of monocytes in mediating neutrophil migration into injured tissues remains unclear. We recently found that donor-derived nonclassical monocytes are necessary for early neutrophil recruitment through the production of the neutrophil chemoattractant MIP-2 and that depletion of these cells ameliorates lung IRI (21). Interestingly, using intravital 2-photon imaging, we have previously observed that circulating monocytes facilitate neutrophil trafficking into freshly reperfused lungs. Furthermore, we found that pulmonary graft function is significantly improved when lung-transplant recipients lack CCR2 expression, which impairs recruitment of classical monocytes to lung grafts $(9,22)$. Since both recruitment to the tissue and extravasation of neutrophils into the extravascular space are necessary for the initiation and propagation of lung IRI (23), it can be postulated that classical monocytes are perhaps essential for neutrophil extravasation. Therefore, we hypothesized that nonclassical and classical monocytes may have complementary roles in the development of IRI, in which nonclassical monocytes provide the initial signals for leukocyte recruitment, while host-derived classical monocytes are essential for the extravasation of neutrophils into the injured lungs. 
$\mathbf{A}$

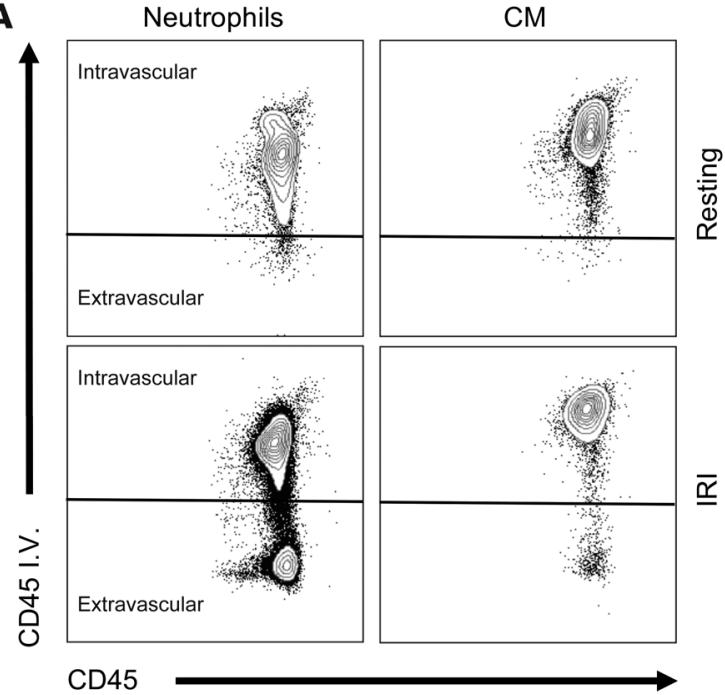

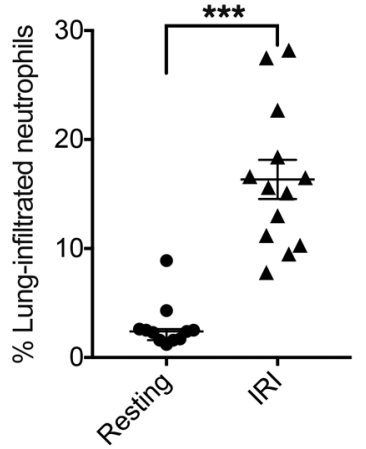

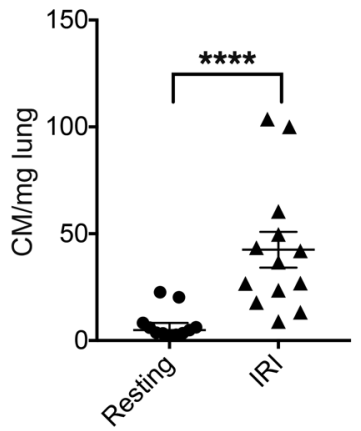

B

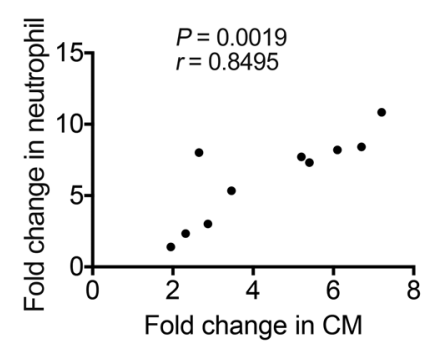

C

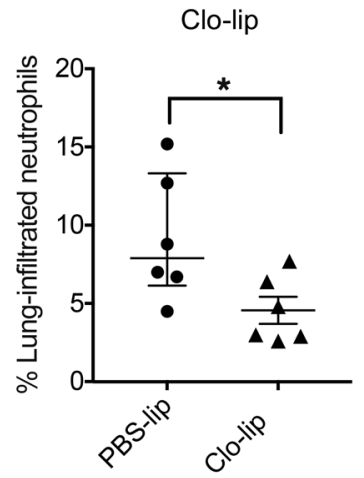

CD11b-DTR

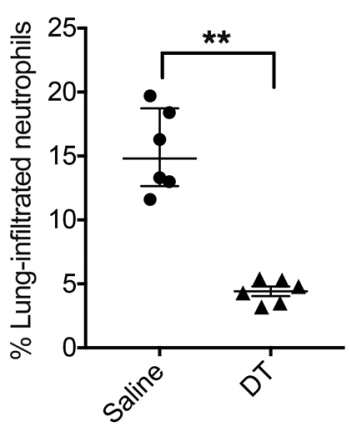

Anti-CCR2 Ab

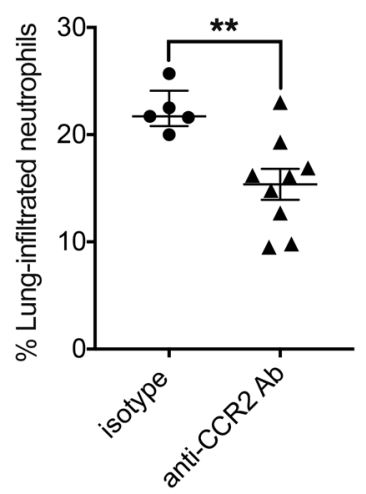

Figure 1. Classical monocytes recruited to the lung after IRI mediate neutrophil extravasation. WT mice underwent IRI using hilar clamping, and the myeloid cell populations were analyzed by flow cytometry. (A) Representative flow cytometry plots (left) and quantification (right) of extravasated neutrophils and recruited classical monocytes (CM) in resting state or that of hilar clamp-mediated IRI are demonstrated. Neutrophils and classical monocytes were gated on live CD45+Ly6C+CD11b+SSChi and live CD45+Ly6C-NK1.1-SiglecF-CD11b+CD64-Ly6Chi cells, respectively. (B) Correlation between recruitment of classical monocytes following reperfusion of human lung allografts and increase in neutrophils in the BALF. The ratio of classical monocytes normalized by alveolar macrophages was correlated with an increase in bronchoalveolar neutrophils before and 15 minutes following reperfusion. Pearson's correlation coefficient $(r$ ) was significant. (C) The percentage of extravasated neutrophils was determined after hilar clamp-mediated IRI in mice that were systemically depleted of monocytes 24 hours prior to IRI using Clo-lip (left), DT (CD11b-DTR mouse; middle), or anti-CCR2 antibody treatment (right). Data are expressed as median with interquartile range. $n=5-13$ per group. ${ }^{*} P<0.05 ;{ }^{*} P<0.01 ;{ }^{* * *} P<0.001 ;{ }^{* * *} P<0.0001$, Mann-Whitney $U$ test $(\mathbf{A}$ and $\mathbf{C})$.

In both human lung allografts and murine models, we found that IRI was associated with the recruitment of classical monocytes as well as neutrophils into the injured lungs. The classical monocytes were mobilized from the host spleen and were necessary for neutrophil extravasation, since pharmacological depletion of $\mathrm{CCR} 2^{+}$classical monocytes as well as splenectomy abrogated neutrophil extravasation, while heterotopic spleen transplantation following initial splenectomy rescued the injury. To achieve clinical relevance, we validated these findings in an orthotopic vascularized murine lung-transplant model (24). We found that host splenectomy ameliorated IRI, while reconstitution with spleen-derived classical monocytes restored IRI. Neutrophil extravasation was mediated by MyD88-dependent IL-1 $\beta$ production by classical monocytes, which resulted in downregulation of $\mathrm{ZO}-2$, a cytoplasmic protein that interacts with transmembrane tight junctional proteins, in pulmonary vascular endothelial cells.
IL-1 $\beta$-mediated downregulation of ZO-2 was sufficient to increase endothelial permeability. Thus, our work uncovers what we believe to be a previously unrecognized mechanism by which classical monocytes mobilized from the spleen promote extravasation of recruited neutrophils into the lung following IRI.

\section{Results}

Classical monocytes are necessary for neutrophil extravasation following lung IRI. We and others have demonstrated that host neutrophils are critical mediators of lung IRI (2, 7-11, 25). Using our previously described technique of multicolor flow cytometry (26-29), we determined the changes in myeloid cell populations in the lung following IRI that was mediated by transient clamping of the pulmonary hilum. We observed that classical monocytes were recruited and host neutrophils extravasated into the lung while the number of nonclassical monocytes remained unchanged after IRI 
A
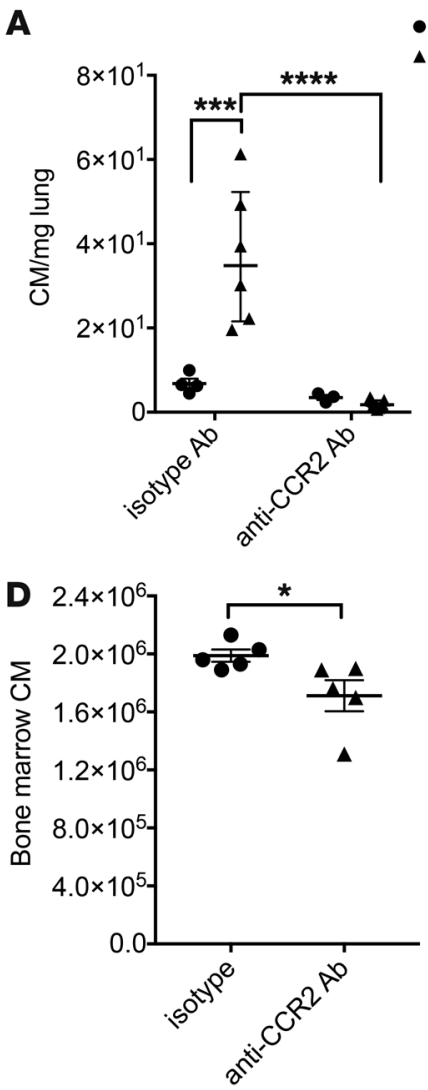

B

$\Delta \mid \mathrm{RI}$

Before IRI

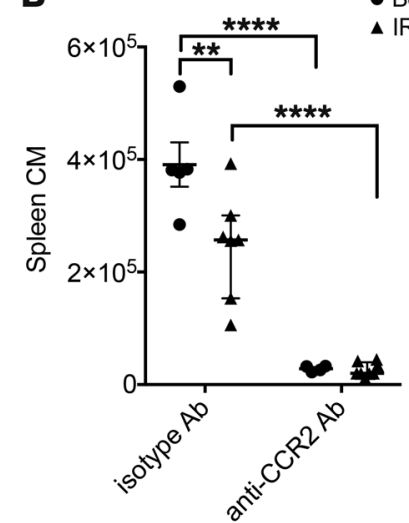

$\mathbf{E}$

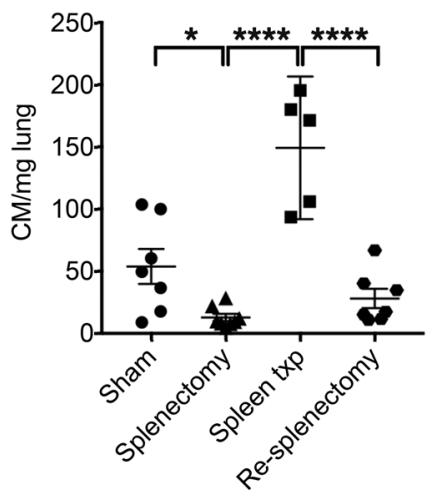

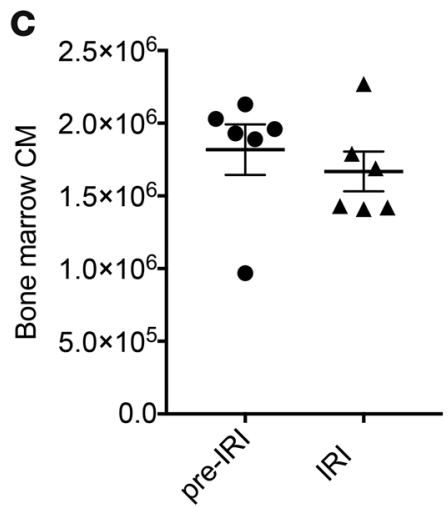

$\mathbf{F}$

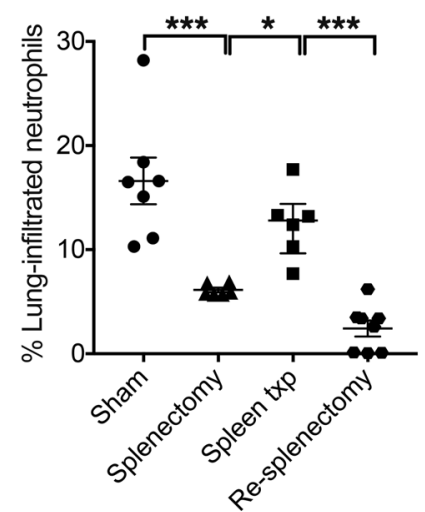

Figure 2. Classical monocytes mediating neutrophil extravasation in lungs subjected to IRI through hilar clamping are mobilized from the spleen. Numbers of classical monocytes in the (A) lung, (B) spleen, and (C) bone marrow before and 2 hours after hilar clamp-mediated lung IRI. Numbers of classical monocytes in the lung and spleen are shown after treatment with isotype control or anti-CCR2 antibody. (D) Numbers of classical monocytes in bone marrow of resting mice after treatment with isotype control or anti-CCR2 antibody. Data are expressed as median with interquartile range. $n=5$ per group. ${ }^{*} P<0.05$, Mann-Whitney $U$ test. (E) Recruitment of classical monocytes to injured lung and $(\mathbf{F})$ percentage of extravasated neutrophils in injured lung after hilar clamp-mediated IRI in the presence or absence of a spleen. Congenic spleen transplants were performed into hosts that had undergone native splenectomies. Splenectomy of the transplanted spleen (resplenectomy) rendered these mice asplenic. txp, transplant. For $\mathbf{E}, 1 \mathrm{statistical}$ outlier in the spleen transplant and 1 statistical outlier in resplenectomy group were excluded from the analysis. For $\mathbf{F}, 1$ statistical outlier was excluded in the splenectomy group. Data are expressed as mean \pm SEM. $n=6$-8 per group. ${ }^{*} P<0.05 ;{ }^{* *} P<0.01 ;{ }^{* *} P<0.001 ;{ }^{* * *} P<0.0001,1$-way ANOVA with post hoc Holm-Šídák test (A-C, E, F).

(Figure 1A, Supplemental Figure 1, and Supplemental Figure 2A; supplemental material available online with this article; https:// doi.org/10.1172/JCI98436DS1). We also observed an increase in neutrophils and classical, but not nonclassical, monocytes in human lung allografts following reperfusion (Supplemental Figure 3). The increase in classical monocytes in human lung grafts correlated with an accumulation of neutrophils in the bronchoalveolar lavage fluid (BALF) (Figure 1B).

In order to determine whether classical monocytes were responsible for neutrophil extravasation, we first depleted all circulating monocytes by pretreating the host with clodronate liposomes (Clo-lip), a technique we have previously demonstrated as causing monocytopenia for at least 72 hours (Supplemental Figure 2B and Supplemental Figure 4) (16). This led to a significant decrease in neutrophil extravasation following lung IRI (Figure 1C). Similarly, we found a significant reduction in neutrophil extravasation in CD11b-DTR mice after monocytes were depleted using diphtheria toxin (DT) (Figure 1C, Supplemental Figure 2C, Supplemental Figure 4, A and B). Since Clo-lip treatment results in depletion of both classical and nonclassical monocytes in WT mice and there exists a possibility that administration of DT could also deplete neutrophils in CD11b-DTR mice, we next treated WT hosts with anti-CCR2 antibodies to selectively deplete classical monocytes (Supplemental Figure 2D and Supplemental Figure 4, A and B), as previously described (21). Anti-CCR2 antibody treatment significantly reduced neutrophil extravasation, indicating that classical monocytes may mediate this process (Figure 1C).

The spleen is necessary for the recruitment of classical monocytes and neutrophil extravasation into the injured lung. Both bone marrow and spleen are sites where monocytes can be recruited from in models of lung inflammation (30). We first set out to analyze classical monocytes in different compartments following lung IRI. While the injured lungs showed an increase in classical monocytes (Figure 2A), levels of classical monocytes were decreased in the host spleen (Figure 2B), but remained constant in the bone marrow before and after injury (Figure 2C). Administration of antiCCR2 antibodies resulted in a significant reduction of classical monocytes in both spleen and bone marrow at baseline (Figure 2, 
A
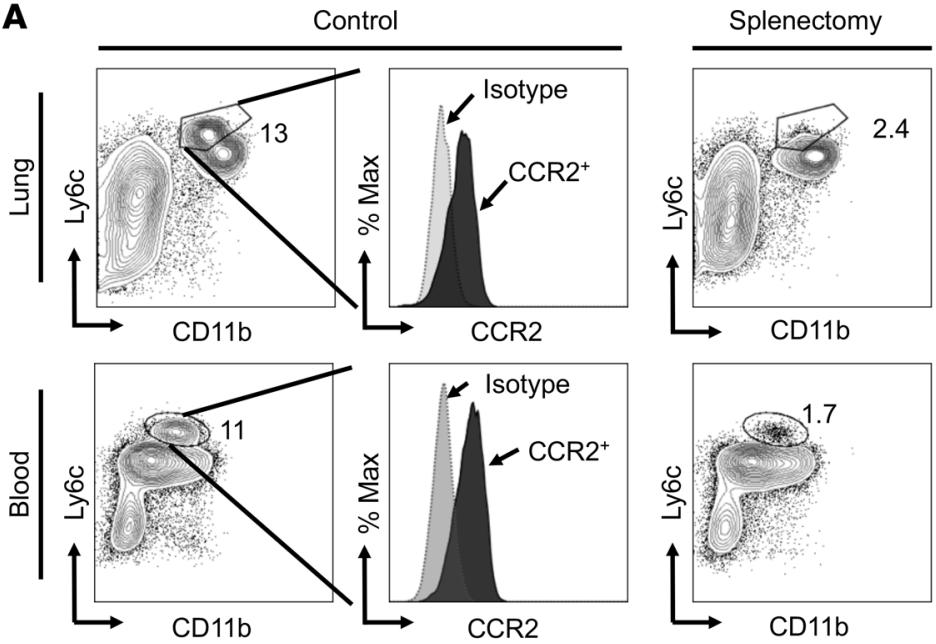

B
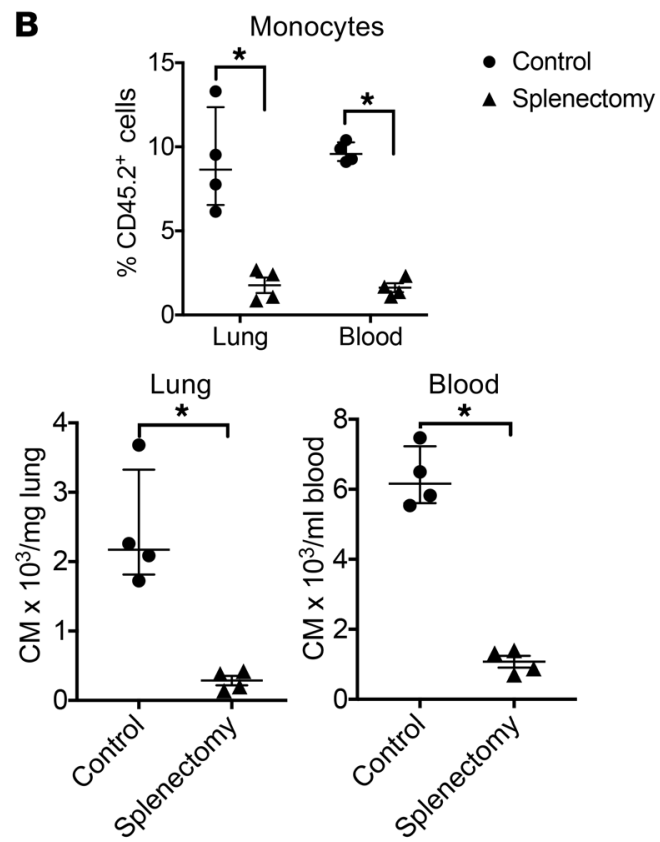

$\mathbf{F}$

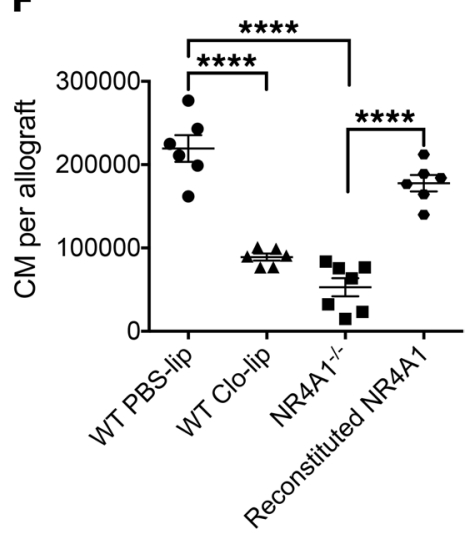

Figure 3. Classical monocytes are mobilized from the recipient's spleen to lung grafts after transplantation. (A) Representative contour plots and histograms depict recipient (CD45.2+) CD11 b+Ly6c ${ }^{\text {hicCR2 }}{ }^{+}$monocytes in pulmonary grafts and peripheral blood 2 hours after transplantation of B6 CD45.1 lungs into control or splenectomized congenic B6 CD45. $2^{+}$mice. (B) Graphic presentation of monocytes as percentages of recipient CD45. $2^{+}$hematopoietic cells and absolute numbers in lung grafts and peripheral blood. Data are expressed as median with interquartile range. $n=4$ per group. ${ }^{*} P<.05$, unpaired $t$ test. (C) A CCR2-specific PET radiotracer ( ${ }^{64} \mathrm{Cu}$-DOTA-ECL1i) was injected into control (left) or splenectomized (right) B6 recipients of syngeneic lung grafts 2 hours after transplantation and imaged by 0 - to 60-minute dynamic PET/CT scanning after intravenous injection. L, liver; $\mathrm{K}$, kidney; B, bladder. Circle shows lung graft. (D) Graphic presentation of ${ }^{64} \mathrm{Cu}$-DOTA-ECL1i uptake in native right lungs and pulmonary grafts 2 hours after transplantation into control or splenectomized syngeneic B6 recipients. Data are expressed as mean \pm SEM. $n=4$ per group. ${ }^{*} P<0.05 ;{ }^{* *} P<0.01 ;{ }^{* * *} P<0.0001,1$-way ANOVA with post hoc Holm-Šídák test. (E) Depletion of nonclassical monocytes in B6 donor lungs through Clo-lip or genetic deletion of NR4A1 led to a reduction in the serum MCP-1 levels of the BALB/c recipient 2 hours after reperfusion compared with control PBS-liposome treatment mice. (F) Recruitment of BALB/c recipient classical monocytes into the $\mathrm{B} 6$ allograft was significantly reduced 2 hours after reperfusion when donor lungs were treated with Clo-lip to deplete donor nonclassical monocytes or when NR4A1-/- donor lungs were used. Reconstitution of B6 NR4A1/-- lungs with WT B6 nonclassical monocytes prior to transplantation restored the levels of classical monocytes recruited to the donor allograft following reperfusion. Data are expressed as mean \pm SEM. $n=5-8$ per group. ${ }^{* * *} P<0.001,1$-way ANOVA with post hoc Holm-Šídák test (E and F).

B and D). After reperfusion, treatment with anti-CCR2 antibody resulted in a significant reduction of classical monocytes in lungs and spleen (Figure 2, A and B). To determine whether classical monocytes can be recruited directly from the bone marrow, we performed a splenectomy, which resulted in a significant reduction in the recruitment of classical monocytes to the injured lungs along with a decrease in neutrophil extravasation, suggesting that the spleen serves as a reservoir for the inflammatory classical monocytes (Figure 2, E and F, and Supplemental Figure 2E). Splenectomy did not alter the number of nonclassical monocytes in injured lungs (Supplemental Figure 2E). In order to further explore this hypothesis, we performed heterotopic congenic spleen transplants (CD45.1 donor into CD45.2 recipient) after native splenectomy, as previously described (31). We found that the donor spleen was repopulated by host-derived myeloid cells and, at 1 week, greater than $95 \%$ of classical monocytes were of recipient origin 
A
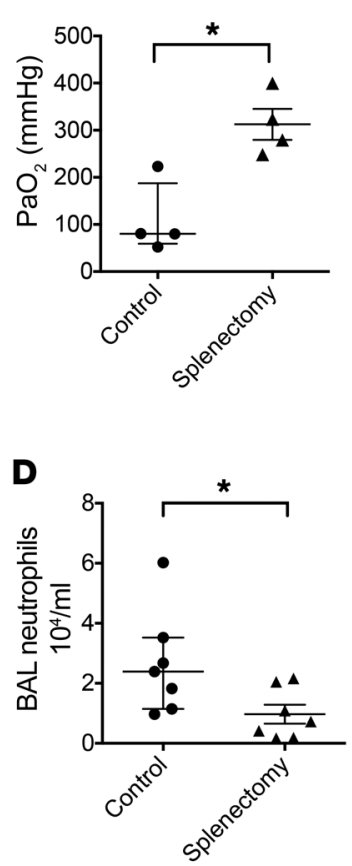

B

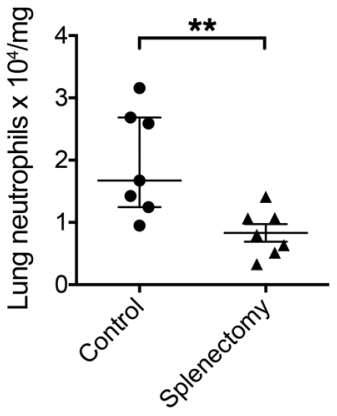

C

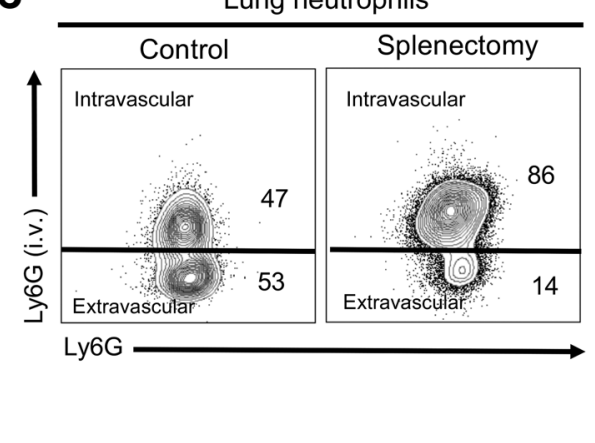

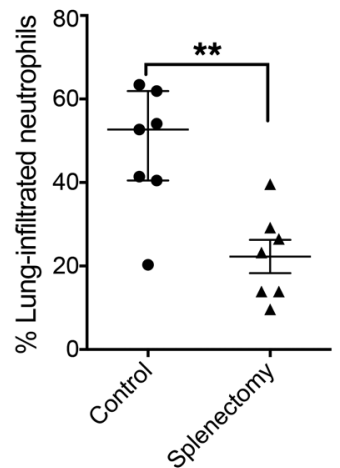

Cxcl5
E

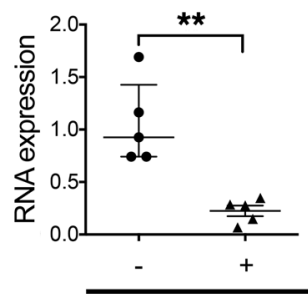

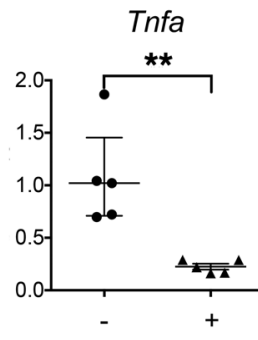
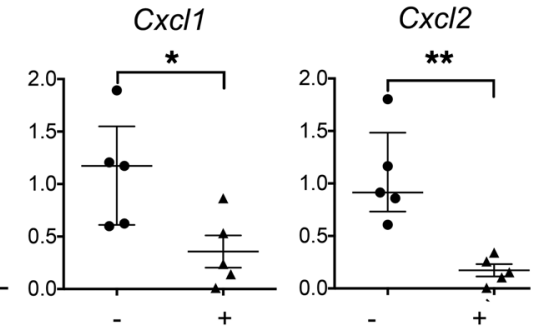

Splenectomy

Figure 4. Spleen-derived classical monocytes promote neutrophil extravasation into lung grafts after transplantation. (A) Arterial blood oxygenation was assessed, and absolute numbers of neutrophils were determined in (B) lung grafts 24 hours after transplantation of B6 CD45.1+ lungs into control or splenectomized congenic B6 CD45.2+ recipients. (C) Contour plots depicting gating strategy to identify intravascular vs. extravascular recipient neutrophils $\left(\mathrm{CD} 45.2^{+} \mathrm{CD} 11 \mathrm{~b}^{+} \mathrm{Gr}-1^{+}\right)$and quantification of the percentage of graft-infiltrating neutrophils 24 hours after transplantation of B6 CD45.1 lungs into control or splenectomized congenic B6 CD45.2+ hosts. Debris, dead cells, and doublets have been excluded from analysis based on forward scatter/side scatter (FSC/ SSC) and FSC-pulse area [FSC-A]/FSC-pulse width [FSC-W] characteristics. (D) Absolute numbers of neutrophils were determined in the BALF 24 hours after transplantation of B6 CD45.1+ lungs into control or splenectomized congenic B6 CD45.2+ recipients. Data for $\mathbf{A}-\mathbf{D}$ are expressed as median with interquartile range. $n=4$ per group for arterial blood gas measurement; $n=7$ per group for enumeration of neutrophils in lung grafts and BALF. ${ }^{*} P<0.05 ;{ }^{* *} P<$ 0.01, Mann-Whitney $U$ test. (E) Expression levels of I/1b, Tnfa, Cxc/1, Cxcl2, and Cxc/5 in B6 CD45.1+ lung grafts 24 hours after transplantation into control or splenectomized congenic B6 CD45.2+ hosts, assessed by quantitative real-time PCR. Results were normalized to Rn18s. Data are expressed as median with interquartile range. $n=5$ per group. ${ }^{*} P<0.05 ;{ }^{* *} P<0.01$, Mann-Whitney $U$ test.

(Supplemental Figure 5). Mice that underwent a native splenectomy and received heterotopic spleen transplants demonstrated preserved recipient-derived classical monocyte infiltration into the lung as well as neutrophil extravasation following pulmonary IRI. Moreover, removal of the transplanted spleen prior to lung IRI abrogated classical monocyte recruitment and neutrophil extravasation, which was similar to our observations in WT mice after splenectomy (Figure 2, E and F). This further confirmed the importance of the spleen as a source of inflammatory classical monocytes that are recruited to injured lungs. Notably, splenectomy did not affect neutrophil extravasation in other models of lung injury, including administration of intratracheal LPS or acid aspiration (Supplemental Figure 6).

Next, to achieve clinical relevance, we used a murine vascularized lung-transplant model to investigate the role of the spleen as a reservoir for inflammatory classical monocytes. Unlike the hilar clamp model, in which the lungs are subjected to warm ischemia, the lung-transplant model mimics the clinical situation in which lungs undergo a period of cold ischemia prior to reperfusion (24). We splenectomized recipient C57BL/6 (B6) CD45.2 mice prior to transplantation of congenic $\mathrm{B} 6 \mathrm{CD} 45.1$ lungs. The abundance of classical monocytes was significantly decreased in the peripheral blood and lung grafts when compared between splenectomized and nonsplenectomized recipients after reperfusion (Figure 3, A and B). Using PET/CT with our recently described CCR2-specific imaging probe, we observed that, after removal of the recipient spleen, the signal in the lung graft was significantly diminished at 2 hours after transplantation, which was similar to our previous observations in CCR2-deficient lung-transplant recipients (Figure 3, C and D) (22). Moreover, there was an overall decrease in PET signal in the periphery of splenectomized hosts. Thus, similarly to what we found in our observations in the hilar clamp model, the spleen is an important reservoir for inflammatory classical monocytes that infiltrate transplanted lungs immediately after reperfusion. Given that we have previously shown that donor nonclassical monocytes mediate the recruitment of recipient neutrophils into pulmonary grafts, we next set out to determine whether nonclassical monocytes played a role in the recruitment of classical monocytes into the transplanted lung (21). At baseline, classical monocytes were more abundant in both bone marrow and spleen compared with nonclassical monocytes (Supplemental Figure 7). However, while more classical monocytes were present in the bone marrow compared with the spleen, we observed more nonclassical monocytes in the spleen 
A

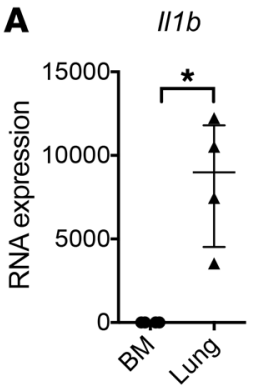

Tnfa

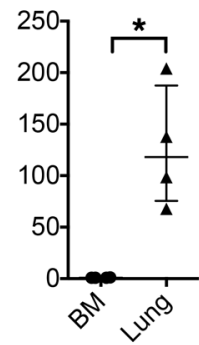

Cxcl1

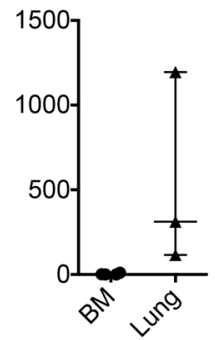

Cxcl2

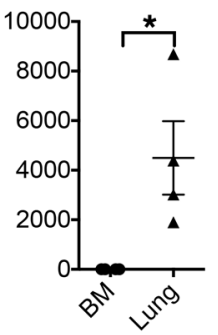

Cxcl5

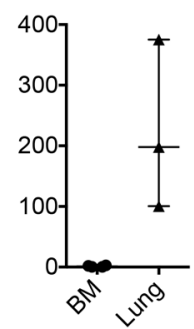

E

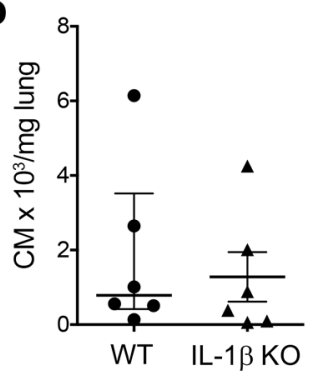

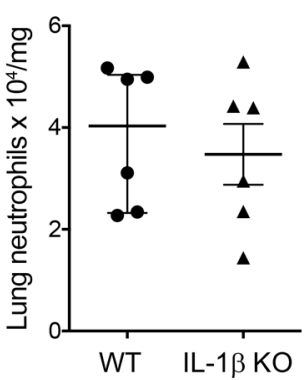

B $\quad I L 1 B$

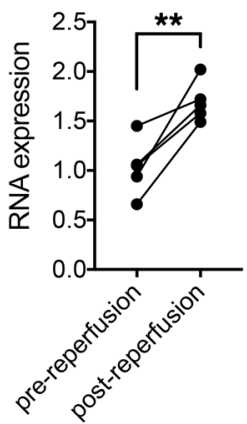

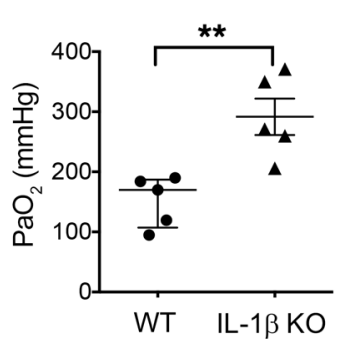

F

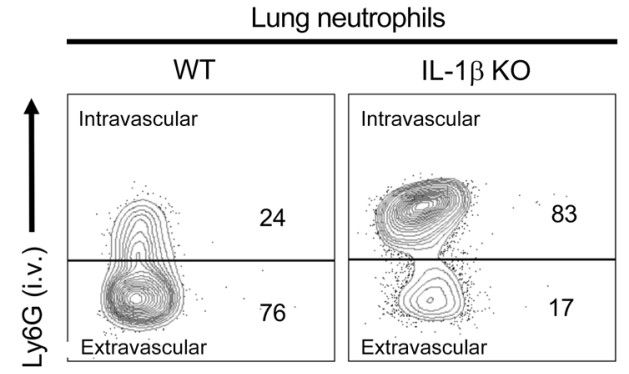

Ly6G

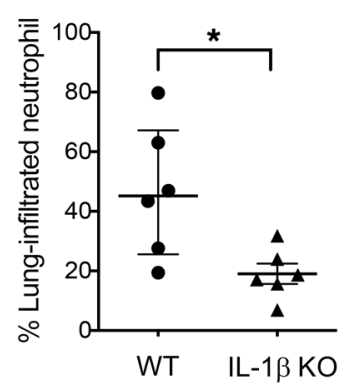

IL1R KO $\rightarrow$ WT
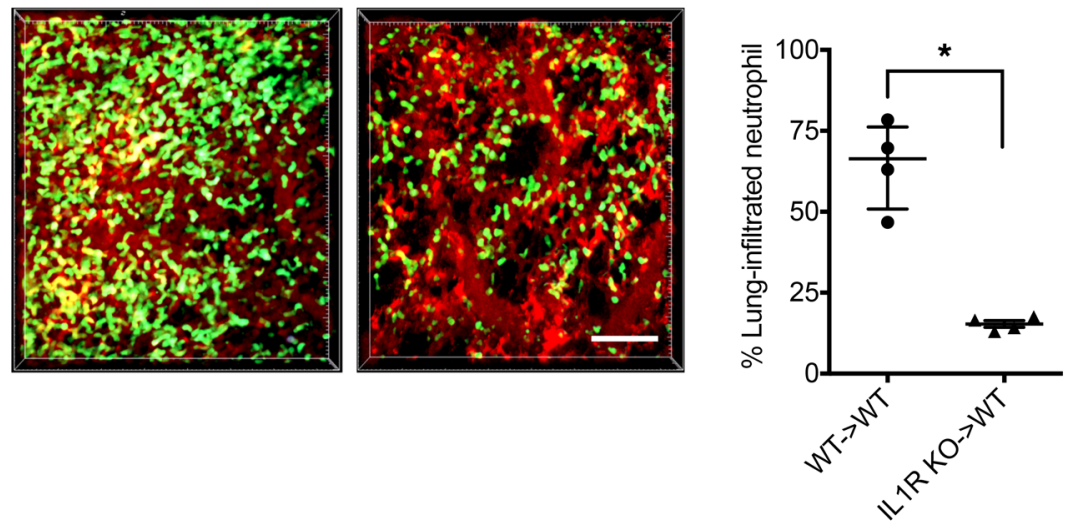

Figure 5. IL-1 $\beta$ production by graft-infiltrating monocytes is essential for neutrophil extravasation after lung transplantation. (A) Recipient monocytes (CD45.2+CD11 $\mathrm{b}^{+} \mathrm{Ly} 6 \mathrm{C}^{\mathrm{hi}}$ ) were isolated from the bone marrow or lung grafts in resting mice or 2 hours after transplantation of B6 CD45.1 lungs into B6 CD45.2 $2^{+}$recipients, and their expression levels of $/ 116$, Tnfa, $C x c / 1, C x c / 2$, and $C x c / 5$ were analyzed by quantitative PCR. $n=4$ each compartment with 1 statistical outlier for $\left[x c / 1\right.$ and 1 statistical outlier for $C x c / 5$ in the lung excluded from analysis. ${ }^{*} P<0.05$, Mann-Whitney $U$ test. (B) Expression levels of IL1B in human donor grafts at conclusion of cold ischemia and 2 hours after reperfusion, assessed by quantitative PCR. $n=5$. ${ }^{* *} P<0.01$, paired $t$ test. (C) Arterial blood oxygenation was assessed and absolute numbers of recipient (D) monocytes and (E) neutrophils were determined in lung grafts 24 hours after transplantation of B6 CD45.1+ lungs into splenectomized congenic B6 CD45.2+ recipients that received either B6 WT or B6 IL-1 $\beta$-deficient monocytes. Data are expressed as median with interquartile range. $n=6$ per group. ${ }^{* *} P<0.01$, Mann-Whitney $U$ test. (F) Representative contour plots of extravascular vs. intravascular neutrophils and quantification of the percentage of graft-infiltrating neutrophils in lung grafts and (C) number of neutrophils in BALF 24 hours after transplantation of B6 CD45.1+ lungs into splenectomized B6 CD45.2+ recipients that received B6 WT or B6 IL-1 $\beta$-deficient monocytes. Data are expressed as median with interquartile range. $n=6$ per group. ${ }^{*} P<0.05$; ${ }^{* *} P<0.01$, Mann-Whitney $U$ test. (H) Representative intravital 2 -photon microscopy of B6 WT or B6 IL-1R-KO lung grafts 2 hours after transplantation into syngeneic B6 LysM-GFP mice and quantification of neutrophil extravasation. Qdot 655 labeled the vessels red. Recipient neutrophils are green. Scale bar: $50 \mu \mathrm{m}$. Data are expressed as median with interquartile range. $n=4$ per group. ${ }^{*} P<0.05$, Mann-Whitney $U$ test. 
compared with the bone marrow (Supplemental Figure 7). We observed that serum levels of the CCR2 ligand MCP-1 were significantly reduced after lung transplantation when grafts were devoid of nonclassical monocytes, which was achieved by pretreating lung donors with Clo-lip injection or by using donors that were deficient in NR4A1, a transcription factor that is necessary for the development of nonclassical monocytes (Figure 3E) (17). Recruitment of classical monocytes was significantly reduced after transplantation of donor lungs that lack nonclassical monocytes (Figure 3F). Importantly, reconstitution of NR4A1-deficient grafts with WT nonclassical monocytes restored the recruitment of classical monocytes to the transplanted lung. Thus, in addition to their previously reported role in mediating neutrophil recruitment, donor nonclassical monocytes promote the recruitment of classical monocytes from the recipient's spleen.

Having shown that spleen-derived classical monocytes infiltrate lung grafts shortly after reperfusion, we next set out to determine whether IRI following lung transplantation is affected by the removal of the recipient's spleen. Compared with what occurred in control recipients, graft function was significantly better in splenectomized hosts (Figure 4A). Since neutrophils play a crucial role in lung IRI, we next compared neutrophil graft infiltration in nonsplenectomized and splenectomized lung recipients. Host splenectomy prior to lung transplantation resulted in an overall reduction of recipient neutrophils in the graft tissue (Figure 4B). Splenectomy resulted in a significant decrease in the percentage of neutrophils that exited the vascular compartment (Figure 4C) and a lower abundance of neutrophils in the airways (Figure 4D), indicating a lower degree of tissue entry and thus supporting the notion that classical monocytes are necessary for the extravasation of host neutrophils following lung IRI. Graft function and neutrophil extravasation in recipients that underwent a sham laparotomy prior to lung transplantation were comparable to that in nonsplenectomized hosts (Supplemental Figure 8, A and B). Furthermore, we observed significantly lower levels of the proinflammatory cytokines $I l 1 b$ and Tnfa (Figure 4E) as well as the neutrophil chemokines $C x c l 1$ and $C x c l 2$, but not $C x c l 5$ transcripts in lung grafts after transplantation into splenectomized hosts (Figure 4E).

Monocyte-derived $I L-1 \beta$ plays a critical role in neutrophil extravasation. In order to elucidate how monocytes regulate neutrophil graft infiltration and their extravasation, we sorted recipient-derived graft-infiltrating classical monocytes and analyzed their gene expression by quantitative reverse-transcription PCR (RT-PCR). Compared with classical monocytes that were concurrently isolated from the bone marrow of these lung-transplant recipients, Il1b expression levels were several thousandfold higher in graft-infiltrating classical monocytes shortly after reperfusion (Figure 5A). Expression levels of Tnfa and $\mathrm{Cxcl} 2$ were also increased in graft-infiltrating monocytes, although they were less pronounced than Il1b (Figure 5A). At baseline, we also observed higher expression of Il1b, Tnfa, and $\mathrm{Cxcl} 2$ in lung compared with bone marrow monocytes, although the differences were less pronounced than after IRI (Supplemental Figure 9). Fractalkine/Cx3cl1 expression could not be detected in monocytes in either compartment (data not shown). Expression levels of $I L 1 B$, but not TNFA, were found to be significantly elevated in human lung grafts after reperfusion
(Figure 5B and Supplemental Figure 10). Based on the markedly elevated levels of IL-1 $\beta$ in graft-infiltrating classical monocytes, we next evaluated whether expression of this cytokine in monocytes regulated neutrophil extravasation into lung grafts during IRI. To this end, we injected splenic classical monocytes isolated from either WT or IL-1 $\beta$-deficient B6 CD45.2 mice into splenectomized B6 CD 45.2 recipients 1 hour prior to transplantation of congenic B6 CD45.1 lungs. Graft function was significantly better after injection of IL-1 $\beta$-deficient compared with WT classical monocytes (Figure 5C). In fact, graft function after adoptive transfer of WT classical monocytes into splenectomized recipients was comparable to that observed after lung transplantation into nonsplenectomized hosts, while graft function after adoptive transfer of IL-1 $\beta$-deficient classical monocytes into splenectomized recipients was not significantly different from that in splenectomized recipients that did not receive monocyte injections (Figure 4A). While the absolute numbers of classical monocytes and neutrophils in lung grafts did not differ between the 2 experimental adoptive transfer groups (Figure 5, D and E), a higher percentage of graft-infiltrating neutrophils had left the vascular compartment (Figure 5F) and a greater number of neutrophils had entered the airways (Figure $5 \mathrm{G}$ ) after adoptive transfer of WT compared with IL-1 $\beta$-deficient classical monocytes. To confirm that IL-1 $\beta$ signaling regulates neutrophil trafficking in freshly reperfused lung grafts, we transplanted lungs that lack expression of the IL-1 receptor (IL-1R) into syngeneic LysM-GFP neutrophil reporter mice and imaged the grafts by intravital 2-photon microscopy 2 hours after reperfusion. Corroborating our flow cytometry data, we observed that, compared with WT grafts, neutrophils failed to extravasate efficiently in IL-1Rdeficient lungs (Figure 5H) (Supplemental Videos 1 and 2). We then compared IL-1 $\beta$ expression in bone marrow and splenic classical monocytes in resting mice and after lung transplantation. Under both conditions, IL-1 $\beta$ expression was significantly increased in splenic monocytes, with a more pronounced difference after lung transplantation (Supplemental Figure 11A). Of note, as opposed to reconstitution with splenic monocytes, injection of bone marrow monocytes into splenectomized hosts did not restore neutrophil extravasation into pulmonary grafts or result in an impairment in oxygen exchange (Supplemental Figure 11, B and C).

MyD88 signaling in graft-infiltrating classical monocytes is necessary for $I L-1 \beta$ production and neutrophil extravasation. We and others have shown that endogenous substances, which are released at the time of organ implantation, can activate innate immune receptors and trigger the release of proinflammatory cytokines, such as IL-1 $\beta$ (32). Many such endogenous triggers of inflammation, referred to as damage-associated molecular patterns, signal via MyD88, an adaptor protein downstream of all TLRs except TLR3 and downstream of IL-1 and IL-18 receptors. We next examined requirements for IL-1 $\beta$ production in monocytes after stimulation with lysates generated from B6 lungs. Expression levels of IL-1 $\beta$ were significantly decreased when monocytes lacked MyD88 (Figure 6A). To assess the role of MyD88 signaling in graft-infiltrating monocytes in vivo, we transferred splenic classical monocytes from either WT or MyD88-deficient B6 CD45.2 mice into splenectomized B6 CD45.2 
A

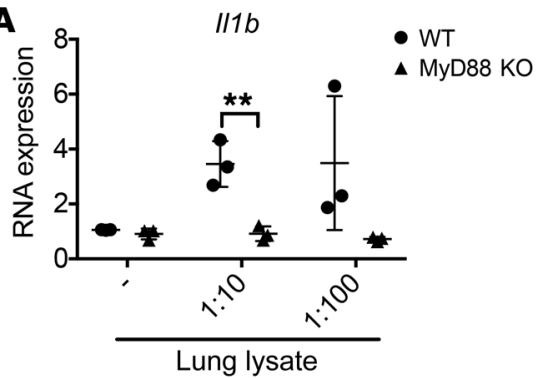

B

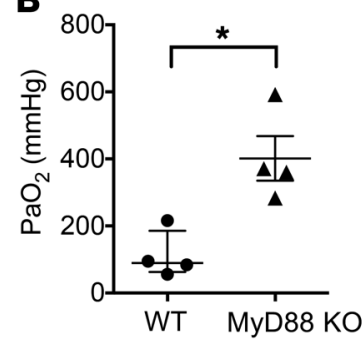

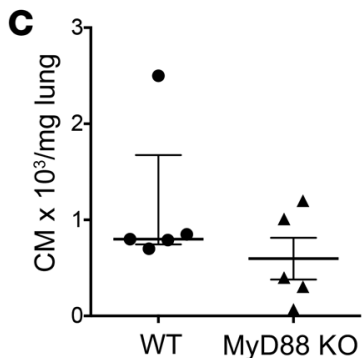

D

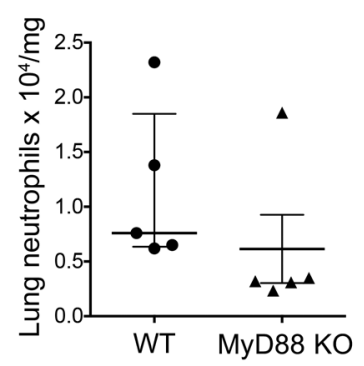

E

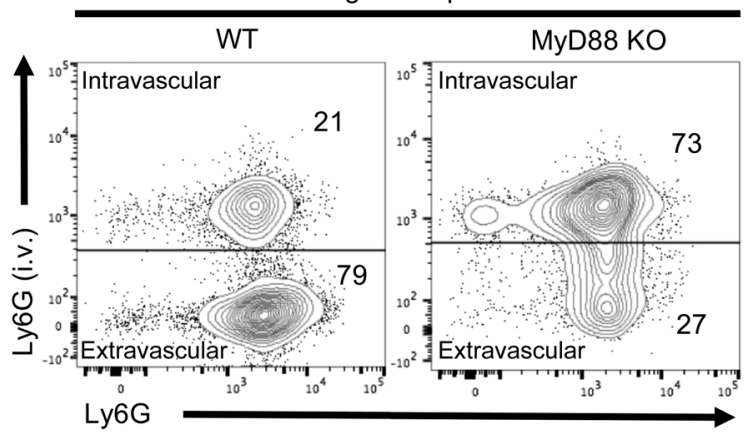

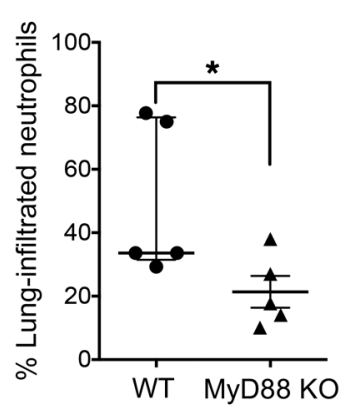

$\mathbf{F}$

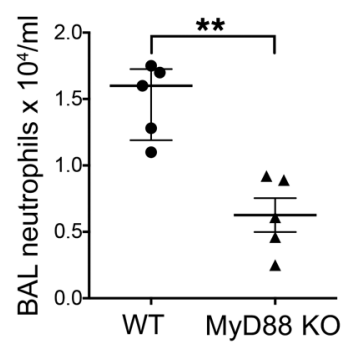

Figure 6. MyD88 expression by graft-infiltrating monocytes is essential for neutrophil extravasation after lung transplantation. (A) Expression levels of IL-1 $\beta$ were assessed by quantitative real-time PCR in bone marrow-derived B6 WT or MyD88-deficient monocytes after treatment with B6 lung lysates. Data are expressed as mean \pm SEM of 3 biological replicates. ${ }^{* *} P<0.01$, unpaired $t$ test. (B) Arterial blood oxygenation was assessed and absolute numbers of recipient (C) monocytes and (D) neutrophils were determined in lung grafts 24 hours after transplantation of B6 CD45.1+ lungs into splenectomized congenic B6 CD45.2+ recipients that received either B6 WT or B6 MyD88-deficient monocytes. (E) Representative contour plots of extravascular vs. intravascular neutrophils and quantification of the percentages of graft-infiltrated neutrophils in lung grafts as well as (F) number of neutrophils in BALF 24 hours after transplantation of B6 CD45.1 $1^{+}$lungs into splenectomized congenic B6 CD45.2 ${ }^{+}$recipients that received either B6 WT or B6 MyD88-deficient monocytes. Data are expressed as median with interquartile range. $n=5$ per group. ${ }^{*} P<0.05 ;{ }^{*} P<0.01$, Mann-Whitey $U$ test (B-F).

hosts 1 hour prior to transplantation of congenic B6 CD45.1 pulmonary grafts. Similarly to our observations with monocytes that lack IL-1 $\beta$, oxygenation was significantly improved after injection of MyD88-deficient when compared with WT monocytes (Figure 6B). The monocyte and neutrophil numbers that had accumulated in the pulmonary grafts were comparable between the 2 experimental groups (Figure 6, C and D); however, a lower percentage of neutrophils extravasated and accumulated in the airways when monocytes lacked expression of MyD88 (Figure 6, E and F).

$I L-1 \beta$ produced by classical monocytes modulates endothelial tight junctions through downregulation of ZO-2. Having shown that neutrophils accumulated in lung grafts, but were unable to exit the vasculature efficiently when graft-infiltrating classical monocytes lacked expression of IL-1 $\beta$ or when IL- $1 \beta$ signaling in the graft was disrupted, we next wanted to determine how endothelial permeability was regulated by IL-1 $1 \beta$. We observed that exposure of primary mouse lung microvascular endothelial cells (MLVECs), grown on Transwell membranes, to IL-1 $\beta$ resulted in a significant increase in their permeability (Figure 7A). This increase in permeability was blunted by pretreating the cultures with an IL-1R-blocking antibody (Figure 7B). To examine downstream mechanisms through which IL-1 $\beta$ disrupts the pulmonary vascular endothelial barrier, we next focused on tight junction proteins. Immunofluorescent staining and Western blotting demonstrated that treatment with IL- $1 \beta$ resulted in a dose-dependent downregulation of $\mathrm{ZO}-2$, a cytoplasmic protein that interacts with transmembrane tight junctional proteins (Figure
7C). Of note, blocking IL-1R signaling attenuated the downregulation of ZO-2 (Figure 7D). We next set out to examine how expression of IL-1 $\beta$ in graft-infiltrating monocytes regulates these processes in vivo. Immunofluorescent staining revealed that the expression of ZO-2 was significantly reduced in $\mathrm{CD} 31^{+}$endothelial cells of $\mathrm{B} 6$ lung grafts that were transplanted into splenectomized syngeneic recipients receiving WT compared with IL-1 $\beta$-deficient monocytes (Figure 7E). We also observed significant downregulation of ZO-2 in human pulmonary grafts following reperfusion (Figure 7F). To elucidate whether downregulation of ZO-2 was sufficient to affect pulmonary endothelial barrier function, an siRNA was used to knock down ZO-2 expression in MLVECs. The knockdown efficiency of the ZO-2 siRNA was confirmed by Western blotting (Figure 8, A and B) and immunofluorescent staining of endothelial cell cultures 48 hours after the transfection (Figure $8 \mathrm{C}$ ). $\mathrm{ZO}-2$ knockdown resulted in enhanced endothelial permeability, as measured by the FITC-dextran assay (Figure 8D) and neutrophil transmigration (Figure 8E).

\section{Discussion}

Lung IRI remains the predominant cause of early allograft failure $(5,6,23,33,34)$. Additionally, it remains the greatest risk factor for the development of chronic lung allograft rejection. Hence, ameliorating lung IRI has the potential for substantially improving pulmonary transplant outcomes, which still remain dismal compared with outcomes for transplants with other solid organs. Host neutrophils are now known to play a central role in the development 
A

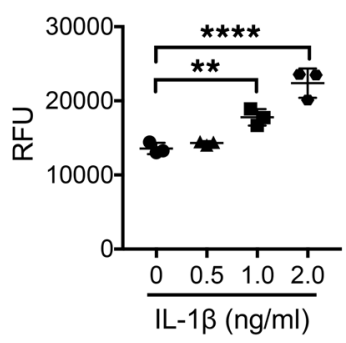

B

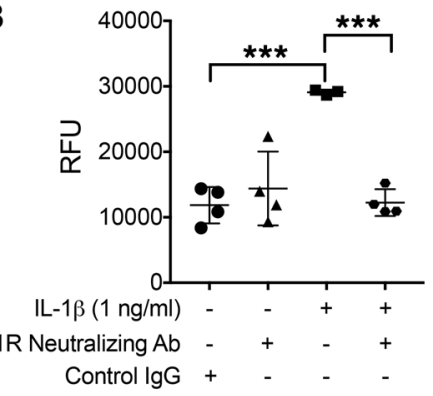

C

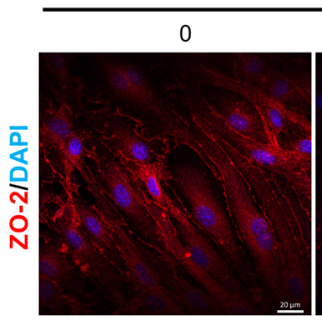

$\mathrm{IL}-1 \beta(\mathrm{ng} / \mathrm{ml})$
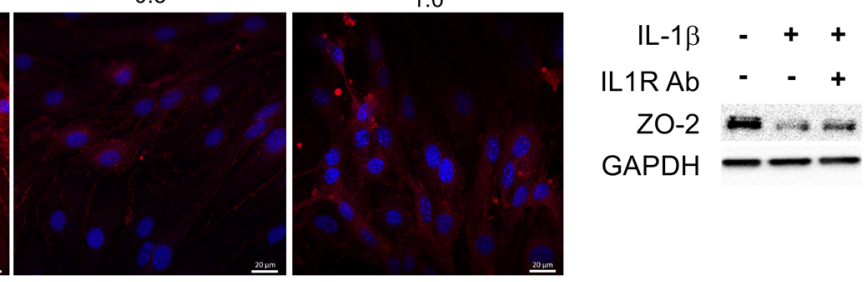

E
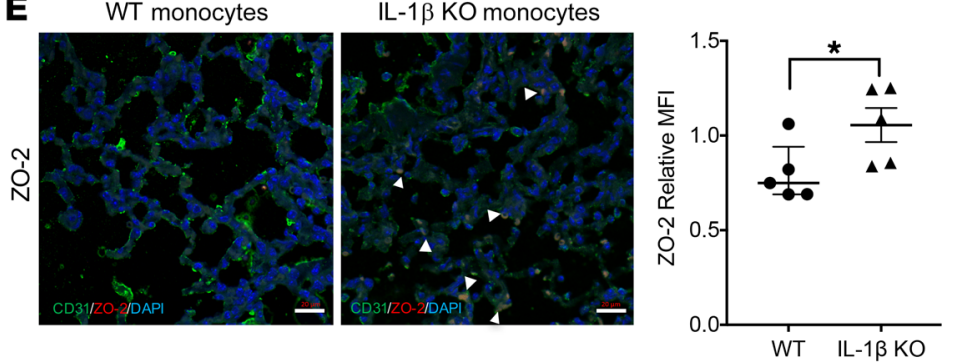

$\mathbf{F}$

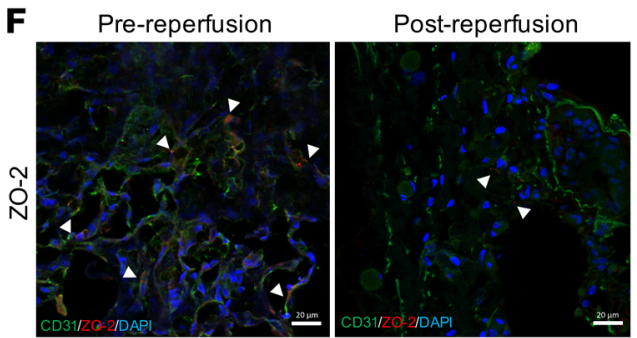

D

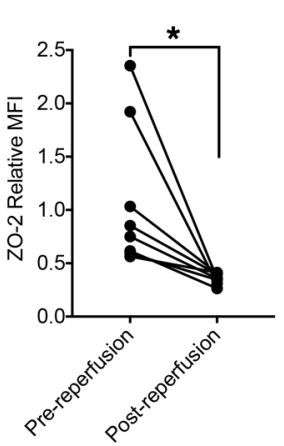

Figure 7. IL-1ß mediates downregulation of ZO-2 in vascular endothelial cells and increases endothelial permeability. Permeability was assessed in MLVEC cultures treated with (A) $\mathrm{IL}-1 \beta$ at indicated concentrations and (B) after adding IL-1R-neutralizing $(2.5 \mu \mathrm{g} / \mathrm{ml})$ or control antibody for 24 hours. For $\mathbf{A}$, a representative experiment of 3 biological replicates is shown. Data represent mean $\pm S D$ of 3 technical replicates. For B, data are presented as mean \pm SD of 4 biological replicates. One statistical outlier was excluded in the group receiving IL-1 $\beta$ treatment only. ${ }^{* *} P<0.01$; ${ }^{* *} P<0.001$; ${ }^{* * *} P<$ 0.001 , 1-way ANOVA with post hoc Holm-Šídák test (A and $\mathbf{B}$ ). RFU, relative fluorescence unit. (C) MLVECs were treated with IL- $1 \beta(0,0.5$, and $1 \mathrm{ng} / \mathrm{ml}$ ) for 24 hours, fixed, and stained with ZO-2. Nuclei were counterstained with DAPI. Images are representative of 3 independent experiments. (D) MLVECs were treated with IL-1R-neutralizing or control antibody for 30 minutes prior to the addition of IL-1 $13(1 \mathrm{ng} / \mathrm{ml})$. Cell lysates were analyzed for ZO-2 by Western blotting. The same blot was reprobed for GAP$\mathrm{DH}$. Blots are representative of 3 independent experiments. (E) Frozen tissues of B6 CD45.1 ${ }^{+}$ lung grafts, collected 24 hours after transplantation into splenectomized B6 CD45.2+ hosts that received B6 WT or B6 IL-1 $\beta$-deficient monocytes and $(\mathbf{F})$ human donor grafts at the conclusion of cold ischemia and 2 hours after reperfusion were stained with antibodies for ZO-2 (red) and CD31 (green). Nuclei were counterstained with DAPI. Arrowhead indicates colocalization of CD31 and ZO-2. Scale bar: $20 \mu \mathrm{m}$. Images were taken with $a \times 40$ objective lens. The relative mean fluorescence intensity (MFI) of ZO-2 staining was quantified and is represented graphically for $\mathbf{E}$ and $\mathbf{F}$. For $\mathbf{E}$, data are expressed as median with interquartile range. $n=5$ per group. ${ }^{*} P<0.05$, Mann-Whitney $U$ test. For $\mathbf{F}, n=8$ patients per group. ${ }^{*} P<0.05$, paired $t$ test. of pulmonary IRI (7-11). Neutrophil trafficking into the injured tissue involves the recruitment of neutrophils to the lung vasculature, followed by extravasation into the interstitial and alveolar space (35). In our recent report, we found that donor nonclassical monocytes provide initial cues for the recruitment of host neutrophils after lung transplantation (21). Here, we demonstrate that extravasation of neutrophils is mediated by host-derived classical monocytes mobilized from the spleen to injured lungs following reperfusion (Figure 9). These classical monocytes produce IL-1 $\beta$ in MyD88-dependent fashion, which plays a critical and nonredundant role in promoting neutrophil extravasation into the lung tissue by acting on the IL-1R on the pulmonary vasculature. IL-1 $\beta$ mediates downregulation of ZO-2 in pulmonary endothelial cells, which we show is sufficient to disrupt the endothelial barrier.
Many studies have demonstrated that CCR2 plays an important role in mediating the egress of inflammatory monocytes from the bone marrow (36). To this end, our group has previously demonstrated that CCR2 is critical for the mobilization of monocytes from the bone marrow into the bloodstream after pulmonary transplantation and for the accumulation of CD11 $\mathrm{c}^{+}$ cells within lung allografts, which enhances alloreactivity (37). While most studies implicating the bone marrow as the source of monocytes examined accumulation of monocyte-derived cells at target sites several days after the inflammatory insult, recent seminal work using models of myocardial ischemia has revealed that the spleen represents the dominant reservoir for monocytes during early stages of inflammation (31). Our findings extend these observations, as we demonstrate by both flow 
A
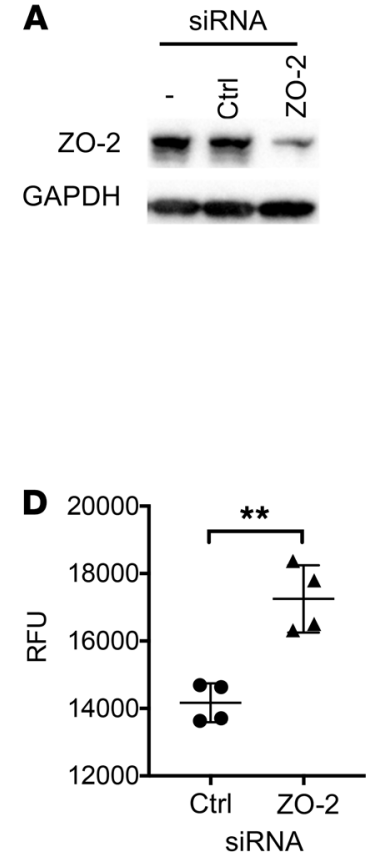

B

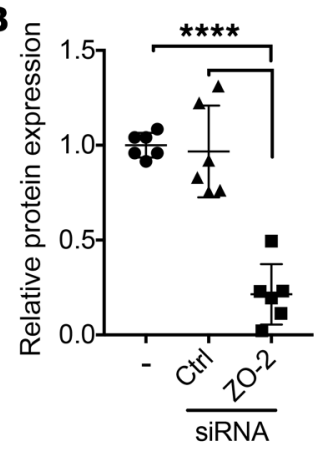

E

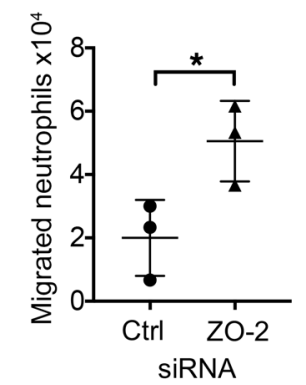

C

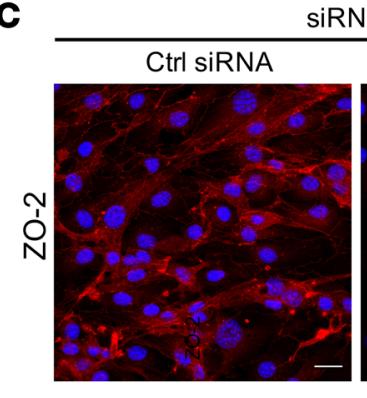

SiRNA

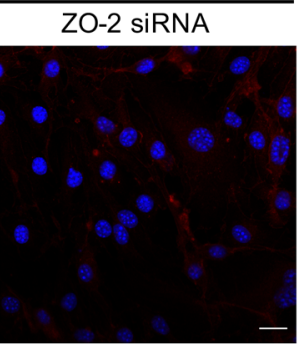

Figure 8. Knockdown of ZO-2 increases endothelial permeability. (A and B) Representative Western blot and semiquantitative analysis as well as (C) immunostaining of ZO-2 in MLVECs that were transfected with control siRNA or siRNA targeting ZO-2 for 48 hours. Scale bars: $20 \mu$ m. Blots and immunostaining are representative of 3 independent experiments each. Data are expressed as mean \pm SD. $n=6$ per group. ${ }^{* * * *} P<0.0001,1$-way ANOVA with post hoc HolmŠídák test. (D) Endothelial permeability was assessed in MLVECs that were treated with control siRNA or siRNA targeting ZO-2, as described in the legend for Figure 7A. Data represent mean \pm SD of 4 biological replicates. ${ }^{*} P<0.001$, unpaired $t$ test. (E) MLVECs were transfected with control siRNA or siRNA targeting ZO-2. Two days later, primary mouse bone marrow-derived neutrophils $\left(1 \times 10^{5}\right.$ cells) were added to the inserts. Twenty-four hours later, transmigrated neutrophils were enumerated in the lower chamber. Data represent mean \pm SD of 3 biological replicates. ${ }^{*} P<0.05$, unpaired $t$ test.

cytometry and PET scanning that CCR2 ${ }^{+}$monocytes are reduced in the periphery and in the pulmonary graft when we remove the recipient's spleen prior to lung IRI. We found that heterotopic spleen grafts were rapidly repopulated by the host classical monocytes, which were then recruited to the injured lung. We further show that spleen monocytes express higher levels of IL-1 $\beta$ at baseline and after transplantation. Hence, it is likely that the spleen serves as a reservoir for bone marrow-derived classical monocytes, where they receive additional maturation signals that enable them to mediate neutrophil extravasation after their rapid release in response to lung injury. We have previously demonstrated that CCR2-deficient lung recipients are protected from transplant-mediated IRI and that neutrophils are the key mediators of IRI after lung transplantation $(22,38)$. Taken together, our findings support the notion that splenectomized lung recipients are protected from IRI through an impairment of monocyte-mediated neutrophil extravasation into the graft. Interestingly, we show that - in contrast with our findings in pulmonary IRI - removal of the spleen does not affect the entry of neutrophils into the lung when injury is triggered through the administration of intratracheal LPS or acid aspiration. We speculate that alveolar macrophages play a dominant role in orchestrating the inflammatory response and neutrophil extravasation when lung injury is triggered through stimuli in the airways. To this end, intratracheal administration of LPS has been shown to induce the death of alveolar macrophages, which results in their release of pro-IL-1 $\alpha$ and vascular leakage (39).
For many years, it had been the prevailing dogma that neutrophils were the first innate cells to enter lung tissue during infections and sterile inflammation. However, the development of approaches to imaging dynamic leukocyte behavior in live lungs allowed us to show that monocyte extravasation temporally precedes and is spatially associated with neutrophil extravasation (9). When we depleted recipient monocytes with intravenous Clo-lip, graft-infiltrating neutrophils adhered to the vessel wall, but their extravasation was impaired at the transendothelial migration step. Disruption of the tight junctions between endothelial cells is a key event that precedes the paracellular passage of leukocytes. Endothelial tight junction integrity is maintained through the interplay of protein complexes. Transmembrane tight junctional proteins, such as claudins, occludins, and junctional adhesion molecules, are anchored to the actin cytoskeleton through cytosolic scaffold proteins such as ZO-1 and ZO-2. Here, we show that monocytic production of IL-1 $\beta$ plays a critical and nonredundant role in increasing endothelial permeability in reperfused lungs. While we cannot exclude the possibility that IL-1 $\beta$ directly alters the expression of other proteins that maintain the functional integrity of the endothelial barrier, we show that downregulation of $\mathrm{ZO}-2$ is sufficient to enhance endothelial permeability. It is surprising that other inflammatory cytokines cannot compensate for IL-1 $\beta$. For example, we have shown that graft-infiltrating monocytes express high levels of TNF- $\alpha$, a cytokine that has been demonstrated to increase the permeability of various endothelial and epithelial cell barriers $(40,41)$. Differences may exist in how tight junctional 


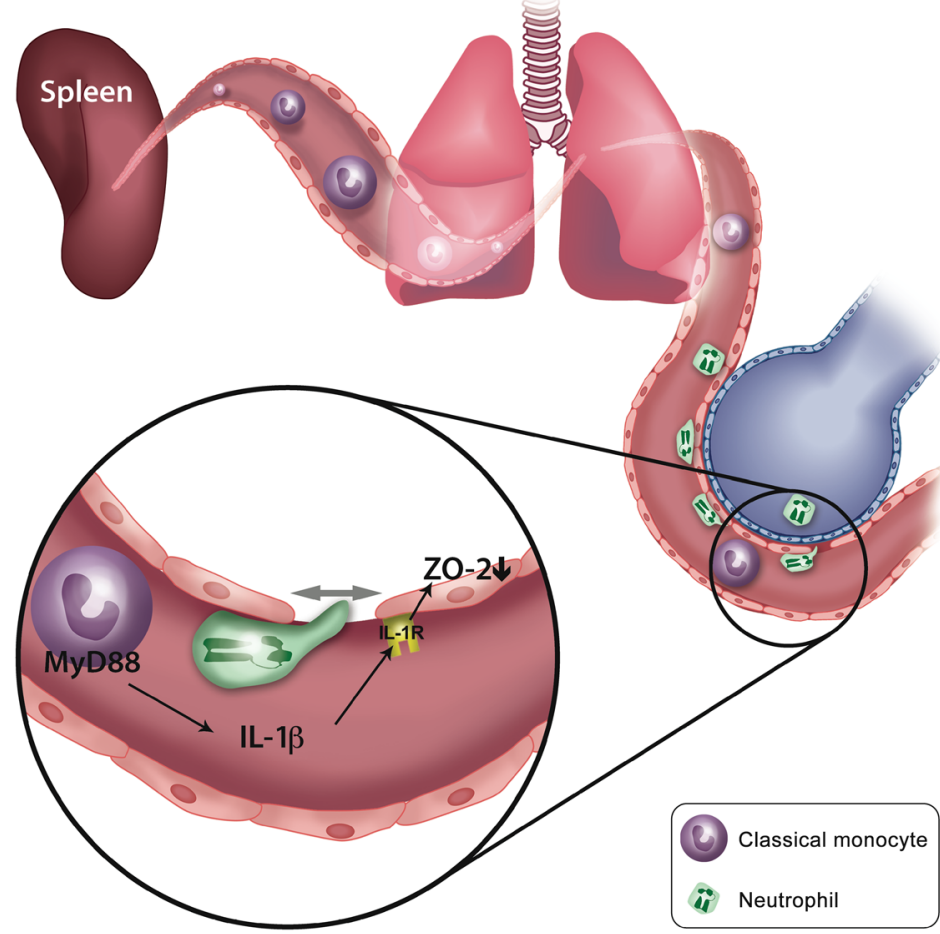

Figure 9. Diagram depicting how spleen-derived classical monocytes mediate extravasation of neutrophils in reperfused lungs via MyD88/IL-1 $\beta$-dependent pathway. Classical monocytes are released from the spleen after reperfusion of lungs. After recruitment to the lung, they produce IL-1 $\beta$ in MyD88-dependent fashion. IL-1 $\beta$ signaling in endothelial cells results in the downregulation of ZO-2, which enhances endothelial permeability and facilitates neutrophil extravasation.

proteins are regulated in various cell types. For example, although IFN- $\gamma$ can disrupt tight junctional proteins in several endothelial and epithelial cells (42), it can enhance the function of the lung epithelial barrier (43).

We speculate that classical monocytes are activated in MyD88-dependent fashion once they enter the reperfused lung due to the release of endogenous substances from injured graft cells. Our laboratory has previously shown that neutrophilic entry into pulmonary graft tissue is impaired when the survival of alveolar macrophages is reduced (44). This defect could be reversed through administration of CXCL2 into the airways. It is possible that, in addition to downregulating tight junctional proteins, graft-infiltrating recipient classical monocytes induce the secretion of neutrophil chemokines by tissue-resident donor cells, such as alveolar macrophages. Collectively, our observations indicate that the production of inflammatory cytokines and chemokines by various cellular sources guides the recruitment of neutrophils into different compartments of the lung. Furthermore, our work establishes a model whereby an interaction between various innate immune cells coordinates the recruitment and extravasation of neutrophils, the cells that ultimately mediate tissue damage during reperfusion of transplanted lungs. This is reminiscent of a recent study that demonstrated that tissue-resident macrophages and inflammatory Ly $6 \mathrm{C}^{\text {hi }}$ monocytes provide cues that guide neutrophils into infected tissue in a murine model of urinary tract infection (45). However, unlike the critical role that we established for monocytic production of IL-1 $\beta$ in pulmonary IRI, neutrophilic entry into bacterially infected urothelium was dependent on the production of TNF- $\alpha$ by monocytes, which induced the release of CXCL2 by macrophages, which in turn facilitated the secretion of MMP-9 by neutrophils.

We have recently shown that a population of lung-resident nonclassical monocytes guides neutrophil recruitment to pulmonary grafts after transplantation through production of the neutrophil chemoattractant CXCL2 (21). Our present study further demonstrates that graft-resident donor nonclassical monocytes mediate secretion of monocyte chemoattractants and play an important role in recruiting recipient spleen-derived classical monocytes to the graft. Importantly, reconstitution of NR4A1-deficient donor lungs with WT syngeneic nonclassical monocytes prior to transplantation restored the recruitment of classical monocytes following reperfusion. Hence, lung-resident nonclassical monocytes might provide the initial signals to recruit both classical monocytes and neutrophils from the periphery, whereas the recruited classical monocytes mediate the extravasation of the recruited neutrophils. We also show that classical monocytes that have been recruited to lung grafts express neutrophil chemokines and may therefore contribute to ongoing neutrophil recruitment and propagation of primary graft dysfunction.

The demonstration that splenic monocyte-derived IL-1 $\beta$ orchestrates critical steps of neutrophil entry into the tissue lays the ground for new therapeutic approaches for lung-transplant recipients and possibly patients suffering from other forms of acute lung injury. While the removal of the spleen is not practical in the clinical setting due to its invasive nature and increased risk for infectious complications, our findings provide a platform for the development of strategies targeting classical monocytes and inhibiting IL-1 $1 \beta$. While no agents that selectively deplete classical monocytes in humans are approved at the present time, several immunosuppressants that are in clinical use have been shown to affect monocyte function. For example, mTOR inhibitors can blunt the expression of chemokines and mycophenolate mofetil can suppress IL-1 $\beta$ production in monocytes in vitro $(46,47)$. Furthermore, as human classical monocytes have been shown to express high levels of CCR2, oral CCR2 inhibitors that are in clinical trials for patients with metastatic pancreatic cancer may be efficacious in ameliorating primary graft dysfunction after lung transplantation (48). Several pharmacological approaches to block the activity of IL-1 $\beta$ are approved for use in the clinic. These include IL-1R antagonists, anti-IL-1 $\beta$ neutralizing antibodies, and soluble decoy receptors. Globally targeting IL-1 $\beta$ has been shown to be efficacious in ameliorating various inflammatory conditions in humans (49). Also, our recently described nanoclusters targeting CCR2 provide a platform to specifically blunt IL-1 production in classical monocytes (22). Previous studies showing monocyte-dependent neutrophil entry in murine arthritis models raise the possibility that analogous mechanisms may regulate sterile inflammation in other organs and tissues (50). 


\section{Methods}

\section{Mice and reagents}

Male B6 (CD45.2+), B6.SJL-PtprcaPepcb/BoyJ (CD45.1+), B6;129S2$\mathrm{Nr} 4 \mathrm{a} 1^{\mathrm{tm} I \mathrm{mi}} / \mathrm{J}$ (Nr4a1 KO), B6.129S7-Il1r1 ${ }^{\mathrm{tm} 1 \mathrm{Imx} / \mathrm{J}}$ (IL-1R KO), B6.FVB-Tg(ITGAM-DTR/EGFP)34Lan/J (CD11b-DTR), BALB/c, and B6 Myd88-deficient (MyD88 KO) mice were purchased from The Jackson Laboratory. B6 LysM-GFP (where LysM indicates lysozyme) mice were originally obtained from Klaus Ley (La Jolla Institute for Allergy and Immunology, La Jolla, California, USA), and B6 IL-1 $\beta$-deficient (IL-1 $\beta$ KO) mice were obtained from Y. Iwakura (Tokyo University of Science, Chiba, Japan, USA) and bred in our facility (51). To deplete intravascular monocytes, mice were injected intravenously with $200 \mu \mathrm{l}$ Clo-lip with PBS-liposomes as control 24 hours prior to lung IRI. Clo-lip and PBS-liposomes were purchased from Liposoma. Cytotoxic anti-CCR2 antibodies (a gift from Steffen Jung, Weizman Institute of Science, Israel) were used for selective depletion of CCR2 ${ }^{+}$classical monocytes as previously described (50 $\mu \mathrm{g}$ intravenously 24 hours prior to IRI) (21). DT (List Biologic Laboratories Inc.) was dissolved in $100 \mu \mathrm{l}$ PBS and administered intraperitoneally to CD11b-DTR mice 24 hours prior to lung IRI. LPS O111:B4 (Sigma Aldrich) was dissolved in PBS and instilled intratracheally at a dose of $2 \mu \mathrm{g} / \mathrm{g}$ with PBS vehicle as control. For acid aspiration injury, $50 \mu \mathrm{l}$ of hydrochloric acid $(0.1 \mathrm{~N})$ was administered into the trachea

\section{Human samples}

Lung graft samples were obtained at the conclusion of cold ischemic storage in Perfadex solution and approximately 120 minutes following reperfusion. A portion of the lung tissue was snap-frozen in liquid nitrogen and subsequently stored at $-80^{\circ} \mathrm{C}$ until the time of processing for gene-expression analysis. A separate portion of lung tissue was fixed in $4 \%$ paraformaldehyde and subsequently processed for immunostaining. Myeloid cell populations were identified from fresh specimens using multicolor flow cytometry with our previously described protocols $(21,26,27)$. For assessment of neutrophil extravasation, the percentage and absolute count of neutrophils were assessed in the BALF prior to lung harvest as well as 15 minutes following reperfusion. Human samples were randomly selected from a cohort of lung-transplant recipients who had consented to participate in these studies, in which graft tissue and BALF were collected before implantation and after reperfusion.

\section{Surgical procedures}

Murine lung transplantation. Orthotopic left lung transplants were performed after 18 hours of cold ischemic storage as previously described $(9,44)$. Graft function was assessed by measuring arterial blood gases while delivering oxygen at an $\mathrm{FiO}_{2}$ of $100 \%$ after clamping the right pulmonary hilum for 4 minutes, as previously described (24). For some experiments, recipients were splenectomized or underwent a sham laparotomy immediately prior to lung transplantation (52). For adoptive transfer experiments, monocytes were isolated from spleens or bone marrow of B6 WT, B6 IL-1 $\beta-\mathrm{KO}$, or B6 MyD88-KO mice using a monocyte isolation kit purchased from Miltenyi Biotec. One hour prior to lung transplantation, $4 \times 10^{5}$ monocytes were injected via penile vein into recipients.

Lung hilar clamping. Mice were anesthetized by intraperitoneal injection of ketamine (100 mg/kg) (Henry Schein Animal Health) and xylazine (10 mg/kg) (RB). Buprenorphine $(0.1 \mathrm{mg} / \mathrm{kg}$ ) (Lloyd Laboratories) was given subcutaneously prior to skin incision. Mice were orotracheally intubated and connected to a rodent ventilator (Harvard Apparatus, model 845) with tidal volumes of $10 \mu \mathrm{l} / \mathrm{g}$ and respiratory rate set at 120 breaths per minute. Left dorsolateral thoracotomy was performed in the fourth intercostal space and a microvascular clamp (Roboz) was applied across the left hilum, occluding the pulmonary artery, vein, and bronchus. While on single-lung ventilation, the tidal volume was reduced to $6 \mu \mathrm{l} / \mathrm{g}$ and respiratory rate increased to 150 breaths per minute. The left hilum was occluded for a 60-minute period, at which point the microvascular clamp was removed. After recruitment of the left lung, the thoracotomy was closed and the mouse was removed from the ventilator and allowed to recover for the 2-hour reperfusion period. For some experiments, mice were splenectomized or underwent sham laparotomy immediately prior to hilar clamping.

Heterotopic spleen transplantation. The heterotopic spleen transplantation procedure was adapted from a previously published technique (31). We used congenic CD45.1 donors and CD45.2 recipients to track donor- and recipient-derived immune cells. In brief, the donor spleen was harvested with an aortic cuff and the portal vein, ligating all arteri$\mathrm{al}$ and venous side branches off the splenic artery and vein. The spleen graft was then implanted in the recipient by anastomosing the donor aortic cuff to the recipient abdominal aorta and the donor portal vein to the recipient inferior vena cava below the renal veins. The recipient underwent a native splenectomy prior to implantation of the spleen graft.

\section{Flow cytometry and cell sorting}

Lung airways were lavaged 5 times with $0.5 \mathrm{ml}$ PBS. BALF was centrifuged, and the cell pellet was processed and prepared for flow cytometric analysis. After the lavage, lung tissue was digested and single cell suspensions were prepared as previously described $(21,27$, 29). Murine bone marrow was harvested from bilateral femurs and tibiae and passed through a $40 \mu \mathrm{m}$ filter to achieve a single cell suspension. Peripheral blood was prepared as previously described (37). Murine spleen was infiltrated with $2.5 \mathrm{ml}$ of digestion buffer $(2 \mathrm{mg} /$ $\mathrm{ml}$ collagenase $\mathrm{D}, 0.2 \mathrm{mg} / \mathrm{ml}$ DNase I, both from Roche, dissolved in HBSS with $\mathrm{Ca}^{2+}$ and $\mathrm{Mn}^{2+}$ ), followed by incubation in an orbital shaker at $37^{\circ} \mathrm{C}$ for 30 minutes, then pushed through a $40 \mu \mathrm{m}$ filter to achieve a single cell suspension. Both bone marrow and spleen suspensions underwent red blood cell lysis using Pharm Lyse buffer (BD Biosciences). Live/dead staining was performed in protein-free solution (HBSS) using fixable viability dye eFluor 506 (eBioscience), followed by incubation with FcR-blocking reagent (Miltenyi Biotec). Cells were then stained using CD45 (clone 30-F11; BD Biosciences), CD45.2 (clone 104; Biolegend), CD45.1 (clone A20; Biolegend), Gr1 (clone RB6-8C5; Biolegend), Ly6C (clone HK1.4; Thermo Fisher Scientific), Ly6G (clone 1A8; Thermo Fisher Scientific), CD11b (clone M1/70; Thermo Fisher Scientific), CCR2 (clone FAB5538A; R\&D System), CD24 (clone M1/69; Thermo Fisher Scientific), I-A/I-E (clone M5/114.15.2; BD Biosciences), CD3 (clone 145-2C11; BD Biosciences), CD4 (clone RM4-5; BD Biosciences), CD8 $\alpha$ (clone 53.6.7; Thermo Fisher Scientific), NK1.1 (clone PK136; Thermo Fisher Scientific), CD64 (clone X54-5/7.1; Biolegend), CD115 (clone AFS98; Thermo Fisher Scientific), SiglecF (clone E50-2440; BD Biosciences), CD19 (clone 1D3; Biolegend), F4/80 (clone BM8; Thermo Fisher Scientific), CD11c (clone HL3; BD Biosciences), CD62L (clone MEL-14; BD Biosciences), CD117 (clone 2B8; Biolegend), and isotype control antibodies (BD Biosciences; BioLegend; and Thermo 
Fisher Scientific). To distinguish between intravascular and interstitial neutrophils, phycoerythrin-labeled (PE-labeled) anti-Ly6G (1A8; eBioscience) was injected intravenously 5 minutes prior to sacrifice, and after digestion, cells were labeled with allophycocyanin-labeled (APC-labeled) anti-Ly6G (1A8; eBioscience), resulting in labeling of intravascular neutrophils as $\mathrm{PE}^{+} \mathrm{APC}^{+}$and interstitial neutrophils as $\mathrm{PE}^{-} \mathrm{APC}^{+}$. In separate experiments, intravascular immune cells were stained with APC-labeled anti-CD45 (30-F11), while the whole tissue digest was stained with FITC-labeled anti-CD45. For some experiments examining gene expression, graft-infiltrating spleen and bone marrow CD90.2-NK1.1-B220- ${ }^{-}{ }^{-} 6 G^{-}{ }^{-}$CD $49 b^{-}{ }^{-C D} 11 b^{+} \mathrm{CD}-$ $11 \mathrm{c}^{\mathrm{lo}} \mathrm{I}-\mathrm{Ab}{ }^{\mathrm{lo}} \mathrm{F} 4 / 80^{\mathrm{lo}} \mathrm{Ly} 6 \mathrm{C}^{\mathrm{hi}}$ monocytes were sorted after staining with antibodies specific for CD115 (c-fms AFS98; Thermo Fisher Scientific), CD11b, Ly6C, CD45.1, and CD45.2 (Beckman Coulter MoFlo). Antibodies used for human cell staining were CD45 (clone H130, eBioscience), CD14 (clone M5E2, eBioscience), HLA-DR (clone L243, eBioscience), CD169 (clone 7-239, BioLegend), CD15 (clone W6D3, BioLegend), CD11b (clone M1/70, BioLegend), CD16 (clone 3G8, eBioscience), CD163 (clone GHI/61, eBioscience), and CD206 (clone 19.2, eBioscience). Fixed samples were run on a custom LSRFortessa Cell Analyzer (eBioscience). Flow cytometry data were analyzed with FlowJo v10 software.

\section{PET/CT}

PET/CT scanning using a CCR2-specific probe was performed as previously described $(22,53)$. Briefly, 2 hours after transplantation, 0 - to 60-minute dynamic PET/CT scanning was performed after the tail vein injection of ${ }^{64} \mathrm{Cu}$-radiolabeled DOTA-ECL1i conjugate $\left({ }^{64} \mathrm{Cu}\right.$-DOTA-ECL1i, 3.7 MBq in $100 \mu \mathrm{l}$ saline) using a cross-calibrated MicroPET Focus 220 (Siemens) or Inveon PET/CT system (Siemens). The tracer uptake was calculated as the percentage of injected dose per gram of tissue in the 3D regions of interest from PET images without partial volume correction using Inveon Research Workplace (Siemens) following this equation: $\mathrm{SUV}=$ radioactivity concentration in ROI $(\mathrm{Bq} /$ $\mathrm{ml}) /($ injected dose $[B q]$ /animal body weight $[g]$ ), where SUV = standardized uptake value, $B q=$ becquerel, and $\mathrm{ROI}=$ region of interest.

The quantitative tracer uptake in the ROI was normalized by the injected radioactivity and animal body weight.

\section{Two-photon microscopy}

Lung grafts were imaged by intravital 2-photon microscopy 2 hours after reperfusion for a time period of 2 hours as previously described $(44,54)$. Qdot 655 (30 $\mu$ l) (Life Technologies) was injected to label vessels. Grafts were exposed and imaged with a custom 2-photon microscope using ImageWarp (A\&B Software). Sequential z-sections (2.5 $\mu \mathrm{m}$ each) were acquired, yielding an imaging volume of $220 \times 247.5$ $\times 50 \mu \mathrm{m}^{3}$. Analyses were performed with Imaris (Bitplane). For each mouse, up to 5 lung areas at least $80 \mu \mathrm{m}$ deep were analyzed $(44,54)$.

\section{Quantitative PCR}

Homogenized lung graft tissue, cultured primary MLVECs (Cell Biologics), and sorted monocytes were lysed with TRIzol (Thermo Fisher Scientific) as previously described (55). Total RNA was isolated using QIAGEN RNeasy Mini Kit (QIAGEN), and quantitative PCR was performed as previously described $(55,56)$. Primer sequences for mouse Tnfa, Cxcl1, Cxcl2, Cxcl5, and Rn18s were previously described (44, 57). Primer sequences for mouse $I l 1 b$ were 5'-GCAACTGTTCCT-
GAACTCAACT-3' and 5'-ATCTTTTGGgGtCCGTCAACT-3'. Primer sequences for human IL1B, TNFA, and RNA18S were as follows: IL1B: 5'-TTCGACACATGGGATAACGAGG-3' and 5'-TTTTTGCTGTGAGTCCCGGAG-3'; TNFA: 5'-CCTGCCCCAATCCCTTTATT-3' and 5'-CCCTAAGCCCCCAATTCTCT-3'; RNA18S: 5'-GGCCCTGTAATTGGAATGAGTC-3' and 5'-CCAAGATCCAACTACGAGCTT-3'.

\section{Cell transfection}

MLVECs were transfected with siRNA targeting ZO-2 (s232643; Thermo Fisher Scientific) or with control siRNA (AM4611: Thermo Fisher Scientific) and cultured for 48 hours prior to analysis. Concentrations of siRNA and transfection reagent (Lipofectamine RNAiMAX; Thermo Fisher Scientific) were selected per the manufacturer's recommendations.

\section{Endothelial permeability assays}

MLVEC monolayer solute permeability was assessed using an in vitro vascular permeability 96-well assay kit (Trevigen). Primary MLVECs $\left(1 \times 10^{5}\right.$, passages $2-4$ were used) were stimulated with various concentrations of IL-1 $\beta$ (R\&D Systems; 401-ML) for 24 hours. Subsequently, monolayer solute permeability was determined by addition of high-molecular weight FITC-Dextran (Trevigen) to the Transwell insert. Twenty-four hours later, the fluorescence intensity in the media from the lower chamber was assessed using Bio-Rad CFX Connect Optical Module. In some experiments, endothelial cell monolayers were treated with IL-1R neutralization antibody ( $\& \& D$ Systems; AF771) or control goat IgG (R\& D Systems; AB-108-C) for 30 minutes prior to adding IL-1 $\beta$. Fluorescence intensity was assessed in triplicate per experimental condition and normalized to untreated control cells. Primary mouse neutrophils were isolated from the bone marrow of WT B6 mice based on a negative selection as described previously (Miltenyi Biotec) (38). MLVECs $\left(1 \times 10^{5}\right)$ were seeded on a gelatin-coated Transwell membrane with a $3 \mu \mathrm{m}$ pore size (Corning Life Science) and cultured for 24 hours. MLVECs were transfected with an siRNA targeting ZO-2 or with a control siRNA for 2 days as outlined above. Neutrophils $\left(1 \times 10^{5}\right)$ suspended in $100 \mu \mathrm{l}$ medium were added to the Transwell insert after 2 washes with PBS and incubated at $37^{\circ} \mathrm{C}$ for 24 hours. The number of transmigrated neutrophils was determined in the lower compartment.

\section{Lung lysate preparation}

Homogenized B6 WT lung samples (KINEMATICA Polytron PT 1200) underwent 5 cycles of freeze-thawing in liquid nitrogen and a $37^{\circ} \mathrm{C}$ water bath. This solution was filtered using a $70 \mu \mathrm{m}$ strainer, and diluted supernatants were used to treat monocytes isolated from the bone marrow of B6 WT or MyD88-KO mice.

\section{Western blotting}

Lysates from endothelial cell cultures were prepared using RIPA buffer containing protease inhibitors (P8340; Sigma-Aldrich) and a phosphatase inhibitor (P0044; Sigma-Aldrich) (55). Protein concentrations were quantitated using a bicinchoninic acid detection assay (Pierce). The lysates were resolved by SDS-PAGE, transferred to nitrocellulose membranes, and probed with antibodies against ZO-2 (Novus Biologicals) and GAPDH (Novus Biologicals) $(55,56)$. The Western blot images were acquired by the ChemiDoc MP image system with Image Lab Software (version 5.1). The band intensities were quantitated by densitometry using AlphaEase imaging software (version 4.0.1). 


\section{Immunostaining}

Frozen lung tissues were prepared and sectioned as previously described (54). MLVECs were fixed with $4 \%$ paraformaldehyde for 15 minutes. Cells were then permeabilized (0.1\% Triton X-100 in PBS for 30 minutes on ice) and blocked. Tissue sections and cells were stained with antibodies against ZO-2, CD31 (clone P2B1; Abcam), Alexa Fluor 647-conjugated CD14 (clone M5E2; Novus Biologicals), CD15 (clone MY-1; Abcam), and PE-conjugated CD16 (clone 3G8; BioLegend). ZO-2 was detected using an Alexa Fluor 555-conjugated secondary antibody, and both CD31 and CD15 were detected using an Alexa Fluor 488-conjugated secondary antibody (Thermo Fisher Scientific). Nuclei were stained with ProLong Gold Antifade Mountant with DAPI (Thermo Fisher Scientific). Images were acquired with a Zeiss LMS 510 Confocal Laser Scanning Microscope or Zeiss Observer Fluorescent Microscope using a $\times 40$ water immersion lens (Zeiss). For both murine and human tissue, intensities of ZO-2 and DAPI staining were quantitated using Zeiss Efficient Navigation software (ZEN; Zeiss). Relative mean fluorescence intensity of ZO-2 and DAPI were analyzed. The intensity of ZO-2 was normalized to that of DAPI. For some experiments, numbers of classical monocytes $\left(\mathrm{CD} 14^{+} \mathrm{CD} 16^{\mathrm{dim}}\right)$, nonclassical monocytes $\left(\mathrm{CD} 14^{+} \mathrm{CD} 16^{+}\right)$, and neutrophils $\left(\mathrm{CD} 15^{+} \mathrm{CD} 16^{+}\right)$were enumerated at $\times 40$ magnification. For all experiments, 5 fields per tissue section were randomly chosen, digitally captured, and analyzed in blinded fashion.

\section{Statistics}

Data were analyzed by Prism version 7.0d. For the comparison of 2 groups, data were analyzed with the Mann-Whitney $U$ test or, when indicated, with 2-tailed paired or unpaired $t$ test or the Pearson's correlation coefficient test, as described for individual experiments. For multiple group comparisons, we tested for normality (Shapiro-Wilks test) and data were analyzed by 1-way ANOVA with post hoc Holm-Šídák test. A $P$ value of less than 0.05 was considered significant. Statistical outliers were determined using the GraphPad outlier calculator using $\alpha=0.05$.

\section{Study approval}

All procedures were approved by the Institutional Animal Studies Committees at Washington University and Northwestern University. Animals received humane care in compliance with the NIH Guide for the Care and Use of Laboratory Animals (National Academies Press, 2011) and the Principles of Laboratory Animal Care formulated by the National Society for Medical Research. Human protocols were approved by the Institutional Review Boards at Washington University School of Medicine (no. 201012829) and Northwestern University Feinberg School of Medicine (no. STU00056197). All study subjects provided informed consent.

\section{Author contributions}

HMH, RF, ST, WL, JHS, SC, MA, DRP, QW, CT, DS, TT, and HL conducted experiments. VP, GRSB, ASK, AVM, KJL, YL, and AEG contributed to study design and reviewed and revised the manuscript. HMH, RF, WL, YL, AB, and DK analyzed and interpreted the data. $\mathrm{HMH}, \mathrm{RF}, \mathrm{AB}$, and $\mathrm{DK}$ wrote the manuscript. $\mathrm{AB}$ and $\mathrm{DK}$ designed and supervised the study.

\section{Acknowledgments}

This work was supported by NIH 1P01AI116501 and R01 HL094601 (to DK and AEG), Veterans Administration Merit Review 1I01BX002730, the Foundation for Barnes-Jewish Hospital (to DK and AEG), the NIH Transplant Scientist Training Program (T32 DK077662 to RF and SC), International Society of Heart and Lung Transplantation Research awards (to $\mathrm{HMH}$ and RF), NIH P01 AG049665, NIH P01 HL071643, Department of the Army W81XWH-15-1-0215 (to GRSB and AVM), and NIH HL125940, the Thoracic Surgery Foundation, the American Lung Association, and the Society of University Surgeons (to AB). The Northwestern University Flow Cytometry Core Facility is supported by a Cancer Center support grant (NCI CA060553). Flow cytometry cell sorting at Northwestern University was performed on a BD FACSAria SORP system, purchased through the support of NIH 1S10OD011996-01. YL and HPL were supported by NIH R01 HL125655 and NIH R01 HL131908. We thank the Molecular Microbiology Imaging Facility at Washington University in St. Louis for their technical support.

Address correspondence to: Daniel Kreisel, Department of Pathology \& Immunology, Campus Box 8234, 660 South Euclid Avenue, Washington University School of Medicine, St. Louis, Missouri 63110, USA. Phone: 314.362.6021; Email: kreiseld@wustl.edu. Or to: Ankit Bharat, Department of Medicine, 676 N. St. Clair Avenue, Suite 650, Northwestern University, Chicago, Illinois 60611, USA. Phone: 312.695.3800; Email: abharat@nm.org.
1. Sørensen OE, Borregaard N. Neutrophil extracellular traps - the dark side of neutrophils. JClin Invest. 2016;126(5):1612-1620.

2. Ley K, Laudanna C, Cybulsky MI, Nourshargh S. Getting to the site of inflammation: the leukocyte adhesion cascade updated. Nat Rev Immunol. 2007;7(9):678-689.

3. Porteous MK, Diamond JM, Christie JD. Primary graft dysfunction: lessons learned about the first $72 \mathrm{~h}$ after lung transplantation. Curr Opin Organ Transplant. 2015;20(5):506-514.

4. Christie JD, et al. Report of the ISHLT Working Group on Primary Lung Graft Dysfunction part II: definition. A consensus statement of the International Society for Heart and Lung Transplantation. J Heart Lung Transplant. 2005;24(10):1454-1459.

5 . Bharat A, et al. Immunological link between primary graft dysfunction and chronic lung allograft rejection. Ann Thorac Surg. 2008;86(1):189-195; discussion 196.

6. Daud SA, et al. Impact of immediate primary lung allograft dysfunction on bronchiolitis obliterans syndrome. Am J Respir Crit Care Med. 2007;175(5):507-513.

7. Belperio JA, et al. CXCR2/CXCR2 ligand biology during lung transplant ischemia-reperfusion injury. JImmunol. 2005;175(10):6931-6939.

8. Zarbock A, Allegretti M, Ley K. Therapeutic inhibition of CXCR2 by Reparixin attenuates acute lung injury in mice. Br J Pharmacol. 2008;155(3):357-364.

9. Kreisel D, et al. In vivo two-photon imaging reveals monocyte-dependent neutrophil extravasation during pulmonary inflammation. Proc Natl Acad Sci U S A. 2010;107(42):18073-18078.

10. Tsai KS, Grayson MH. Pulmonary defense mech- anisms against pneumonia and sepsis. Curr Opin Pulm Med. 2008;14(3):260-265.

11. Tate MD, Deng YM, Jones JE, Anderson GP, Brooks AG, Reading PC. Neutrophils ameliorate lung injury and the development of severe disease during influenza infection. JImmunol. 2009;183(11):7441-7450.

12. Ziegler-Heitbrock L, et al. Nomenclature of monocytes and dendritic cells in blood. Blood. 2010;116(16):e74-e80.

13. Chiu S, Bharat A. Role of monocytes and macrophages in regulating immune response following lung transplantation. Curr Opin Organ Transplant. 2016;21(3):239-245.

14. Hanna RN, et al. Patrolling monocytes control tumor metastasis to the lung. Science. 2015;350(6263):985-990.

15. Cros J, et al. Human CD14dim monocytes patrol 
and sense nucleic acids and viruses via TLR7 and TLR8 receptors. Immunity. 2010;33(3):375-386.

16. Misharin AV, et al. Nonclassical Ly6C(-) monocytes drive the development of inflammatory arthritis in mice. Cell Rep. 2014;9(2):591-604.

17. Carlin LM, et al. Nr4a1-dependent Ly6C(low) monocytes monitor endothelial cells and orchestrate their disposal. Cell. 2013;153(2):362-375.

18. Collison JL, Carlin LM, Eichmann M, Geissmann F, Peakman M. Heterogeneity in the locomotory behavior of human monocyte subsets over human vascular endothelium in vitro. J Immunol. 2015;195(3):1162-1170.

19. Auffray C, et al. Monitoring of blood vessels and tissues by a population of monocytes with patrolling behavior. Science. 2007;317(5838):666-670.

20. Nahrendorf $\mathrm{M}$, et al. The healing myocardium sequentially mobilizes two monocyte subsets with divergent and complementary functions. JExp Med. 2007;204(12):3037-3047.

21. Zheng $Z$, et al. Donor pulmonary intravascular nonclassical monocytes recruit recipient neutrophils and mediate primary lung allograft dysfunction. Sci Transl Med. 2017;9(394):eaal4508.

22. Liu $\mathrm{Y}$, et al. Noninvasive imaging of CCR2+ cells in ischemia-reperfusion injury after lung transplantation. Am J Transplant. 2016;16(10):3016-3023.

23. Sayah DM, et al. Neutrophil extracellular traps are pathogenic in primary graft dysfunction after lung transplantation. Am J Respir Crit Care Med. 2015;191(4):455-463.

24. Okazaki M, et al. A mouse model of orthotopic vascularized aerated lung transplantation. Am J Transplant. 2007;7(6):1672-1679.

25. Belperio JA, et al. Critical role for CXCR2 and CXCR2 ligands during the pathogenesis of ventilator-induced lung injury. J Clin Invest. 2002;110(11):1703-1716.

26. Misharin AV, et al. Monocyte-derived alveolar macrophages drive lung fibrosis and persist in the lung over the life span. J Exp Med. 2017;214(8):2387-2404.

27. Bharat A, et al. Flow cytometry reveals similarities between lung macrophages in humans and mice. Am J Respir Cell Mol Biol. 2016;54(1):147-149.

28. Desch AN, et al. Flow cytometric analysis of mononuclear phagocytes in nondiseased human lung and lung-draining lymph nodes. Am J Respir Crit Care Med. 2016;193(6):614-626.

29. Misharin AV, Morales-Nebreda L, Mutlu GM, Budinger GR, Perlman H. Flow cytometric analysis of macrophages and dendritic cell subsets in the mouse lung. Am J Respir Cell Mol Biol.
2013;49(4):503-510.

30. Francis M, et al. Editor's Highlight: role of spleen-derived macrophages in ozone-induced lung inflammation and injury. Toxicol Sci. 2017;155(1):182-195.

31. Swirski FK, et al. Identification of splenic reservoir monocytes and their deployment to inflammatory sites. Science. 2009;325(5940):612-616.

32. Mori DN, Kreisel D, Fullerton JN, Gilroy DW, Goldstein DR. Inflammatory triggers of acute rejection of organ allografts. Immunol Rev. 2014;258(1):132-144.

33. Bharat A, et al. Antibodies to self-antigens predispose to primary lung allograft dysfunction and chronic rejection. Ann Thorac Surg. 2010;90(4):1094-1101.

34. Hachem RR. Lung allograft rejection: diagnosis and management. Curr Opin Organ Transplant. 2009;14(5):477-482.

35. Martin TR. Neutrophils and lung injury: getting it right. J Clin Invest. 2002;110(11):1603-1605.

36. Serbina NV, Pamer EG. Monocyte emigration from bone marrow during bacterial infection requires signals mediated by chemokine receptor CCR2. Nat Immunol. 2006;7(3):311-317.

37. Gelman AE, et al. CCR2 regulates monocyte recruitment as well as $\mathrm{CD} 4 \mathrm{~T} 1$ allorecognition after lung transplantation. Am J Transplant. 2010;10(5):1189-1199.

38. Kreisel D, et al. Bcl3 prevents acute inflammatory lung injury in mice by restraining emergency granulopoiesis. J Clin Invest. 2011;121(1):265-276.

39. Dagvadorj J, et al. Lipopolysaccharide induces alveolar macrophage necrosis via CD14 and the $\mathrm{P} 2 \mathrm{X} 7$ receptor leading to interleukin-1 $\alpha$ release. Immunity. 2015;42(4):640-653.

40. Baddoura FK, Nasr IW, Wrobel B, Li Q, Ruddle $\mathrm{NH}$, Lakkis FG. Lymphoid neogenesis in murine cardiac allografts undergoing chronic rejection. Am J Transplant. 2005;5(3):510-516.

41. Mehta D, Malik AB. Signaling mechanisms regulating endothelial permeability. Physiol Rev. 2006;86(1):279-367.

42. Bruewer M, Utech M, Ivanov AI, Hopkins AM, Parkos CA, Nusrat A. Interferon-gamma induces internalization of epithelial tight junction proteins via a macropinocytosis-like process. FASEB J. 2005;19(8):923-933.

43. Ahdieh M, Vandenbos T, Youakim A. Lung epithelial barrier function and wound healing are decreased by IL- 4 and IL-13 and enhanced by IFN-gamma. Am J Physiol, Cell Physiol. 2001;281(6):C2029-C2038.
44. Spahn JH, et al. DAP12 expression in lung macrophages mediates ischemia/reperfusion injury by promoting neutrophil extravasation. JImmunol. 2015;194(8):4039-4048.

45. Schiwon M, et al. Crosstalk between sentinel and helper macrophages permits neutrophil migration into infected uroepithelium. Cell. 2014;156(3):456-468.

46. Lin HY, et al. Effects of the mTOR inhibitor rapamycin on monocyte-secreted chemokines. $B M C$ Immunol. 2014;15:37.

47. Weimer R, et al. Mycophenolate mofetil-based immunosuppression and cytokine genotypes: effects on monokine secretion and antigen presentation in long-term renal transplant recipients. Transplantation. 2003;75(12):2090-2099.

48. Thomas GD, et al. Human blood monocyte subsets: a new gating strategy defined using cell surface markers identified by mass cytometry. Arterioscler Thromb Vasc Biol. 2017;37(8):1548-1558.

49. Ridker PM, et al. Antiinflammatory therapy with canakinumab for atherosclerotic disease. $N$ Engl J Med. 2017;377(12):1119-1131.

50. Shimada K, et al. Oxidized mitochondrial DNA activates the NLRP 3 inflammasome during apoptosis. Immunity. 2012;36(3):401-414.

51. Horai R, et al. Production of mice deficient in genes for interleukin (IL)-1alpha, IL-1beta, IL-1alpha/beta, and IL-1 receptor antagonist shows that IL-1beta is crucial in turpentineinduced fever development and glucocorticoid secretion. J Exp Med.1998;187(9):1463-1475.

52. Huston JM, et al. Splenectomy protects against sepsis lethality and reduces serum HMGB1 levels. JImmunol. 2008;181(5):3535-3539.

53. Liu Y, et al. PET-based imaging of chemokine receptor 2 in experimental and disease-related lung inflammation. Radiology. 2017;283(3):758-768.

54. Koltsova EK, et al. Dynamic T cell-APC interactions sustain chronic inflammation in atherosclerosis. J Clin Invest. 2012;122(9):3114-3126.

55. Hsiao HM, Thatcher TH, Colas RA, Serhan CN, Phipps RP, Sime PJ. Resolvin D1 reduces emphysema and chronic inflammation. Am J Pathol. 2015;185(12):3189-3201.

56. Hsiao HM, et al. A novel anti-inflammatory and pro-resolving role for resolvin D1 in acute cigarette smoke-induced lung inflammation. PLoS One. 2013;8(3):e58258.

57. Li W, et al. Heart-resident CCR2+ macrophages promote neutrophil extravasation through TLR9/MyD88/CXCL5 signaling. JCI Insight. 2016;1(12):e87315. 